\title{
An efficient local coupled cluster method for accurate thermochemistry of large systems
}

\author{
Hans-Joachim Werner ${ }^{1, a)}$ and Martin Schütz ${ }^{2, b)}$ \\ ${ }^{1}$ Institut für Theoretische Chemie, Universität Stuttgart, Pfaffenwaldring 55, \\ D-70569 Stuttgart, Germany \\ ${ }^{2}$ Institut für Physikalische und Theoretische Chemie, Universität Regensburg, Universitätsstr. 31, \\ D-93040 Regensburg, Germany
}

(Received 12 July 2011; accepted 31 August 2011; published online 13 October 2011)

\begin{abstract}
An efficient local coupled cluster method with single and double excitation operators and perturbative treatment of triple excitations [DF-LCCSD(T)] is described. All required two-electron integrals are evaluated using density fitting approximations. These have a negligible effect on the accuracy but reduce the computational effort by 1-2 orders of magnitude, as compared to standard integral-direct methods. Excitations are restricted to local subsets of non-orthogonal virtual orbitals (domain approximation). Depending on distance criteria, the correlated electron pairs are classified into strong, close, weak, and very distant pairs. Only strong pairs, which typically account for more than $90 \%$ of the correlation energy, are optimized in the LCCSD treatment. The remaining close and weak pairs are approximated by LMP2 (local second-order Møller-Plesset perturbation theory); very distant pairs are neglected. It is demonstrated that the accuracy of this scheme can be significantly improved by including the close pair LMP2 amplitudes in the LCCSD equations, as well as in the perturbative treatment of the triples excitations. Using this ansatz for the wavefunction, the evaluation and transformation of the two-electron integrals scale cubically with molecular size. If local density fitting approximations are activated, this is reduced to linear scaling. The LCCSD iterations scale quadratically, but linear scaling can be achieved by neglecting some terms involving contractions of single excitations. The accuracy and efficiency of the method is systematically tested using various approximations, and calculations for molecules with up to 90 atoms and 2636 basis functions are presented. (C) 2011 American Institute of Physics. [doi:10.1063/1.3641642]
\end{abstract}

\section{INTRODUCTION}

The coupled cluster method with single and double excitations and a perturbative treatment of triple excitations $[\operatorname{CCSD}(\mathrm{T})]$ has become the "Gold Standard" in quantum chemistry (this expression is attributed to T. H. Dunning). Provided that the Hartree-Fock reference function is a good zeroth-order approximation and large basis sets are used, CCSD(T) usually yields energy differences, such as reaction energies with chemical accuracy $(1 \mathrm{kcal} / \mathrm{mol}$ or better), and also many other molecular properties can be accurately predicted. However, there are two bottlenecks that restrict the applicability of the $\operatorname{CCSD}(\mathrm{T})$ method to very small molecules: First, the computational increases with $\mathcal{O}\left(\mathcal{N}^{7}\right)$, where $\mathcal{N}$ is a measure of the molecular size or the number of correlated electrons. This causes a "scaling wall" that cannot be overcome even with massively parallel supercomputers. Second, the convergence of the correlation energy with respect to the basis set size is very slow, and very large basis sets are needed to obtain accurate results. The latter problem can be much alleviated by adding terms to the wavefunction that depend explicitly on the inter-electronic distances $r_{12}$ (socalled F12-methods). This will be addressed in the companion paper, ${ }^{1}$ in which the current work is extended to include such terms.

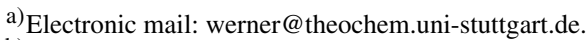

b) Electronic mail: martin.schuetz@ chemie.uni-regensburg.de.
}

The scaling problem can be overcome by exploiting the fact that electron correlation in insulators is a short-range effect. If a local orbital basis is used, approximations that exploit the short-range character can be introduced, as first proposed and implemented by Pulay and Saeb $\varnothing,{ }^{2-6}$ and later generalized and extended in our groups. ${ }^{7-23}$ These methods are based on two distinct approximations: first, excitations from a given pair of localized molecular orbitals (LMOs) are restricted to a subspace (domain) of non-orthogonal projected atomic orbitals (PAOs), which are spatially close to the two LMOs. Second, the pairs of LMOs can be classified according to their distance. Strong pairs, for which the two orbitals are very close, are treated at the highest level, e.g., $\operatorname{LCCSD}(\mathrm{T})$, and more distant weak pairs at lower level, e.g., LMP2. Very distant pairs can be neglected. This has made it possible to achieve linear scaling of the computational cost with molecular size for the whole range of closed-shell single reference methods. ${ }^{9-13,16}$ Based on the techniques described in this paper, also an open-shell DF-LUCCSD(T0) method has recently been developed, which will be described in Refs. 24 and 25. Furthermore, another variant, denoted OSV-LCCSD (local coupled-cluster with orbital specific virtual orbitals), has recently been implemented into our program. ${ }^{26}$ This is based on tensor factorizations of the MP2 amplitudes. ${ }^{27}$ There are also many other methods that exploit the locality of electron correlation, ${ }^{28-40}$ but a review of all these methods is beyond the scope of the current article. 
Our first linear scaling LCCSD implementation ${ }^{9}$ was based on a semi-direct scheme. The integrals in the LMO/PAO basis involving at most three PAOs were generated once and stored on disk (for simplicity, we will in the following denote the integral classes with 1-4 PAOs as 1external to 4-external integrals, respectively). However, the contributions of the 4-external integrals were accounted for in an integral-direct manner by transforming in each iteration the pair amplitude matrices into the $\mathrm{AO}$ basis and contracting them with the integrals computed on the fly. Linear scaling was achieved by pre-screening techniques. Despite the asymptotic linear scaling property, this approach had several disadvantages: first, the 2-electron integrals in the AO basis had to be computed in each iteration. Second, the transformation of the amplitude matrices from the pair specific PAO to the full AO basis reduces the sparsity and, therefore, the contraction with the 2-electron integrals was expensive. Third, the efficiency of the integral screening techniques, which rely on the sparsity of certain test densities, depends sensitively on the structure of the molecule and the basis set. For molecules with a compact three-dimensional structure low-order scaling was reached only for very large systems, in particular, if basis sets with diffuse functions are used. Thus, calculations for realistic molecules with good basis sets were still extremely expensive.

Somewhat later, this problem was alleviated by a semidirect implementation, ${ }^{13}$ in which also the 4-external integrals were transformed into the PAO basis and stored on disk. The number of transformed integrals scales linearly with molecular size, and the contraction with the amplitudes is then very fast. The bottleneck of this implementation is the integraldirect transformation, which is very expensive, in particular when large and diffuse basis sets are used.

The evaluation and transformation of the two-electron integrals can be very much accelerated by using density fitting (DF) approximations. The DF approximations are widely used in electronic structure theory. Originating from density functional theory (DFT) (Refs. 41-43) their usage spreads today from $\mathrm{HF},{ }^{44-46} \mathrm{MP} 2,{ }^{14,47-50} \mathrm{CC} 2,{ }^{20,51} \mathrm{MP} 2-\mathrm{F} 12,{ }^{52-55}$ up to CCSD. ${ }^{16,56}$ Furthermore, for correlated calculations of periodic systems DF is indispensable for an efficient method. ${ }^{57,58}$ The overall scaling of the computational effort with respect to system size is not affected by standard DF, but it significantly reduces the prefactor. Furthermore, for some methods such as MP2 it reduces the scaling with respect to the basis set size per atom, $N_{\mathrm{AO}} / N_{\text {atoms }}$, from quartic to cubic. This leads to enormous savings in calculations with large basis sets. If density fitting is used with localized orbitals, domains can also be introduced for the auxiliary basis set (local fitting), and then linear scaling can be achieved. This was first shown for the calculation of the exchange contribution in local MP2 (Ref. 14) and later extended to other methods. ${ }^{15,16,46,59-61}$ The errors caused by density fitting approximations are generally very small and systematic, in particular for energy differences.

As a first step towards a fully density fitted DFLCCSD(T) implementation, a linear scaling DF algorithm to evaluate the 4-external integrals in LCCSD was presented already in $2003 .{ }^{16}$ In the current work, this approach is extended to treat all integral classes by density fitting. It will be shown that overall this speeds up $\operatorname{LCCSD}(T)$ calculations by 1-2 orders of magnitude, in particular, when large basis sets are used. For simplicity, the prefix DF will be omitted in the following.

A preliminary (unpublished) version of the current method has been available in MOLPRO (Refs. 85 and 86) since some time. In the current work we describe the method in detail, including recent enhancements, such as accelerated LCCSD convergence, improved local density fitting approximations, improved accuracy of weak pair approximations, and more efficient approximations for treating the 3-external integrals. Furthermore, we propose MP2 corrections for the domain and basis set incompleteness errors.

The paper is organized as follows: in Sec. II some basic concepts of LCCSD theory will be reviewed. In Sec. III, the density fitting algorithms for the transformation of the various integral classes will be discussed. Finally, illustrative examples and timings will be presented in Sec. IV.

\section{THEORY}

The LCCSD equations have been presented in full detail by Hampel and Werner. ${ }^{7}$ Further aspects that are important for achieving linear scaling of the computational cost with molecular size have been discussed in our previous work. ${ }^{9}$ However, the formulation used in these papers differs from the present one in some details as explained below, and we will therefore briefly review the method as currently used in our program.

\section{A. Local orbital spaces}

In order to introduce local approximations as required to achieve low-order scaling of the computational cost with molecular size, local orbitals have to be used. Orthonormal occupied localized orbitals (LMOs) can be obtained by standard localization schemes, such has Foster-Boys ${ }^{62}$ or PipekMezey localization. ${ }^{63}$ Alternatively, natural localized orbitals (NLMOs) (Ref. 64) can be employed, which have been found to be less dependent on the basis set. ${ }^{18}$ The virtual orbital space is more difficult to localize by unitary transformations, and, therefore, non-orthogonal virtual orbitals are often generated. In the present work, we use PAOs, ${ }^{2}$ but other choices are possible as well: for example, Neese et al. ${ }^{37}$ have successfully used pair natural orbitals (PNOs), and Yang et al. ${ }^{27}$ proposed to use orbital specific virtuals (OSVs), obtained by tensor factorizations of MP2 amplitudes. The latter method has recently been implemented into our program and will be reported in Ref. 26. The PNO and OSV methods have the advantage that a smaller number of virtual orbitals is required to represent the wavefunction, but since the total number of pair- or orbital-specific orbitals is much larger than the number of PAOs, the integral transformations are more difficult. Furthermore, it is not yet clear how to treat triple excitations with these schemes. Very recently, Jasík et al. have proposed a new method to generate a single set of orthonormal virtual orbitals ${ }^{65}$ that are supposed to be better localized than PAOs, 
but these orbitals have not yet been used in local coupledcluster methods.

In the following, the indices $i, j, \ldots$ refer to LMOs, $r, s$ refer to PAOs, $a, b$ refer to canonical virtual orbitals (VMOs), and $p, q$ refer to any orbitals. The PAOs are defined as

$$
\left|\phi_{r}^{\mathrm{PAO}}\right\rangle=\sum_{a}^{\text {virt }}\left|\phi_{a}\right\rangle\left\langle\phi_{a} \mid \phi_{r}^{\mathrm{AO}}\right\rangle=\sum_{a}^{\text {virt }}\left|\phi_{a}\right\rangle Q_{a r},
$$

where $\left|\phi_{r}^{\mathrm{AO}}\right\rangle$ are atomic orbitals. These are projected onto the virtual orbital space, such that the PAOs are orthogonal on all LMOs, $\left\langle\phi_{r}^{\mathrm{PAO}} \mid \phi_{i}^{\mathrm{LMO}}\right\rangle=0$ for all $i, r$. We assume that the AOs and VMOs are expanded in a common orbital basis set (OBS) $\left\{\chi_{\mu}\right\}$ and represented by coefficient matrices $C_{\mu r}^{\mathrm{AO}}$ and $C_{\mu a}^{\mathrm{v}}$, respectively. The matrix $Q_{a r}$ that transforms from the canonical virtual orbitals to the PAOs can then be written as

$$
\mathbf{Q}=\mathbf{C}^{\mathrm{v} \dagger} \mathbf{S C}{ }^{\mathrm{AO}}
$$

where $S_{\mu \nu}=\left\langle\chi_{\mu} \mid \chi_{\nu}\right\rangle$ is the overlap matrix of the OBS. Thus, the coefficient matrix of the PAOs is

$$
\mathbf{P}=\mathbf{C}^{\mathrm{v}} \mathbf{Q}=\mathbf{C}^{\mathrm{v}} \mathbf{C}^{\mathrm{v} \dagger} \mathbf{S} \mathbf{C}^{\mathrm{AO}} .
$$

The choice of the AOs $\left|\phi_{r}^{\mathrm{AO}}\right\rangle$ is, in principle, arbitrary. For numerical reasons, it is recommended that the PAOs resulting from inner-shell core orbitals are eliminated from the beginning, since their norm would be almost zero if the corresponding LMOs strongly overlap with the AOs. In order to make the selection of core AOs unique, a good general choice is to use for each atom the canonical Hartree-Fock AOs, represented only by the basis functions of the corresponding atom, so that $\mathbf{C}^{\mathrm{AO}}$ is a block-diagonal matrix. In case that correlation consistent or atomic natural orbital basis sets are used, the contracted basis functions $\chi_{\mu}$ automatically correspond either to atomic core or valence orbitals or to correlation functions and, therefore, one can in these cases simply choose $\mathbf{C}^{\mathrm{AO}}$ to be the unit matrix. Optionally, one can normalize the PAOs; then $\mathrm{C}^{\mathrm{AO}}$ becomes a diagonal matrix containing the normalization factors. Since we will only use correlation consistent basis sets, $\mathbf{C}^{\mathrm{AO}}$ is assumed to be diagonal from now on.

\section{B. The local CCSD equations}

For non-metallic systems, the PAOs are local by definition. This makes it possible to introduce domain approximations: the single excitations from a LMO $\phi_{i}$ are restricted to a subset of PAOs $\phi_{r}^{\mathrm{PAO}}, r \in[i]$, which are spatially close to the LMO $\phi_{i}$. The subsets [ $\left.i\right]$ are denoted as orbital domains. Correspondingly, the double excitations from a pair of LMOs $\phi_{i}$, $\phi_{j}$ are restricted to a pair domain $[i j]$, which is the union of the orbital domains $[i]$ and $[j]$. The definition of the domains will be further discussed in Sec. II C. Furthermore, pair approximations can be introduced, and only the amplitudes of strong pairs are optimized in the LCCSD. The pair approximations will be discussed in more detail in Sec. II D.

Using these approximations, the LCCSD wavefunction can be defined as

$$
\Psi=\exp (\hat{T})|0\rangle
$$

with the cluster operator,

$$
\hat{T}=\sum_{i} \sum_{r \in[i]} \hat{E}_{i}^{r} t_{r}^{i}+\frac{1}{2} \sum_{i j \in P_{s}} \sum_{r, s \in[i j]} \hat{E}_{i}^{r} \hat{E}_{j}^{s} T_{r s}^{i j} .
$$

Here $|0\rangle$ is the closed-shell Hartree-Fock reference function, $\hat{E}_{i}^{r}$ are spin-conserving one-particle excitation operators, and $t_{r}^{i}$ and $T_{r s}^{i j}$ are the singles and doubles amplitudes, respectively. The index ranges $r \in[i]$ and $r, s \in[i j]$ are confined to the corresponding domains. The list of orbital pairs $i j$ in the second summation is restricted to a subset $P_{s}$ of strong pairs (possibly also close pairs are included, cf. Sec. II D). Since for a given pair $i j$ the excitation operators $\hat{E}_{i}^{r}$ and $\hat{E}_{j}^{s}$ commute, the doubles amplitude must satisfy the symmetry relation $T_{r s}^{i j}=T_{s r}^{j i}$. For convenience in later expressions, the redundant contributions $i j$ and $j i(i \neq j)$ are both included in the summations.

A compact and computationally convenient form of LCCSD amplitude equations is obtained by multiplying the Schrödinger equation from the left by $e^{-\hat{T}}$ and projecting with the contravariant configurations, ${ }^{66,67}$

$$
\begin{gathered}
\tilde{\Phi}_{i}^{r}=\frac{1}{2} \hat{E}_{i}^{r}|0\rangle, \\
\tilde{\Phi}_{i j}^{r s}=\frac{1}{6}\left(2 \hat{E}_{i}^{r} \hat{E}_{j}^{s}+\hat{E}_{i}^{s} \hat{E}_{j}^{r}\right)|0\rangle .
\end{gathered}
$$

This yields the linked CC residuals

$$
\begin{gathered}
r_{r}^{i}=\left\langle\tilde{\Phi}_{i}^{r}\left|e^{-\hat{T}} \hat{H} e^{\hat{T}}\right| 0\right\rangle, \\
R_{r s}^{i j}=\left\langle\tilde{\Phi}_{i j}^{r s}\left|e^{-\hat{T}} \hat{H} e^{\hat{T}}\right| 0\right\rangle,
\end{gathered}
$$

which must vanish for the desired solution, i.e., $r_{r}^{i}=0$ for $r \in[i]$ and $R_{r s}^{i j}=0$ for $r, s \in[i j]$ (note that the orbital domains $[i]$ equal the diagonal pair domains [ii] and, therefore, do not need a special treatment). The explicit form of the residuals is most easily derived first in the canonical MO basis and then transformed into the PAO basis. They differ from the CCSD equations in the canonical virtual orbital basis by multiplications with the PAO overlap matrix $\mathbf{S}_{\mathrm{PAO}}=\mathbf{Q}^{\dagger} \mathbf{Q}$ in all places where an amplitude index is not matched by an integral index. We note that the current formulation of the LCCSD equations differs from the one given in Ref. 7, which was based on the unlinked CC equations (cf. Appendix A). The full equations as used in the current work are given in Appendix B.

In each LCCSD iteration, the residuals are used to determine updates for the amplitudes. For this purpose, it is necessary to transform them into a pair-specific orthonormal basis,

$$
\bar{\phi}_{a}^{i j}=\sum_{r \in[i j]} \phi_{r}^{\mathrm{PAO}} W_{r a}^{i j}
$$

where the transformation matrices $W_{r a}^{i j}$ are obtained by solving the Fock equations in the domain $[i j]$ as

$$
\mathbf{F}_{\mathrm{PAO}}^{i j} \mathbf{W}^{i j}=\mathbf{S}_{\mathrm{PAO}}^{i j} \mathbf{W}^{i j} \boldsymbol{\Lambda}^{i j}
$$

with $\Lambda_{a b}^{i j}=\delta_{a b} \epsilon_{a}^{i j}$. Here, $\mathbf{F}_{\mathrm{PAO}}^{i j}$ and $\mathbf{S}_{\mathrm{PAO}}^{i j}$ are the Fock and overlap matrices in the PAO basis for the domain $[i j]$. Since 
the full set of PAOs is linearly dependent, it may happen that the PAOs in a domain are (nearly) linearly dependent. Redundant functions are eliminated at this stage by removing the eigenvectors of $\mathbf{S}_{\mathrm{PAO}}^{i j}$ that correspond to zero or very small eigenvalues (singular value decomposition; the default value for the threshold is 1.d-7). Note that for this purpose $\mathbf{S}_{\mathrm{PAO}}^{i j}$ is represented in the unnormalized PAO basis (obtained with $\mathbf{C}^{\mathrm{AO}}=\mathbf{1}$ ), so that contributions of PAOs with a small norm are eliminated first.

In each LCCSD macroiteration, updates of the amplitudes are obtained by solving the LMP2-like linear equations,

$$
\begin{aligned}
0 & =\mathbf{r}^{i}+\mathbf{F} \Delta \mathbf{t}^{i}-\sum_{k} f_{i k} \mathbf{S} \Delta \mathbf{t}^{k}, \\
0= & \mathbf{R}^{i j}+\mathbf{F} \Delta \mathbf{T}^{i j} \mathbf{S}+\mathbf{S} \Delta \mathbf{T}^{i j} \mathbf{F} \\
& -\sum_{k} \mathbf{S}\left[f_{i k} \Delta \mathbf{T}^{k j}+\Delta \mathbf{T}^{i k} f_{k j}\right] \mathbf{S} .
\end{aligned}
$$

Using Eqs. (10) and (11), these equations can be solved iteratively (microiterations) by transforming them to the pairspecific orthogonal basis. In each microiteration, amplitude updates are calculated as

$$
\begin{gathered}
\Delta \bar{t}_{a}^{i}=-\frac{\bar{r}_{a}^{i}}{\epsilon_{a}^{(i i)}-f_{i i}}, \\
\Delta \bar{T}_{a b}^{i j}=-\frac{\bar{R}_{a b}^{i j}}{\epsilon_{a}^{i j}+\epsilon_{b}^{i j}-f_{i i}-f_{j j}},
\end{gathered}
$$

where $f_{i i}$ are the diagonal elements of the Fock matrix in the LMO basis and

$$
\begin{gathered}
\overline{\mathbf{r}}^{i}=\left[\mathbf{r}^{i}-\sum_{k \neq i} f_{i k} \mathbf{S} \Delta \mathbf{t}^{k}\right] \mathbf{W}^{i i}, \\
\overline{\mathbf{R}}^{i j}=\mathbf{W}^{i j \dagger}\left[\mathbf{R}^{i j}-\sum_{k \neq i} f_{i k} \mathbf{S} \Delta \mathbf{T}^{k j} \mathbf{S}-\sum_{k \neq j} \mathbf{S} \Delta \mathbf{T}^{i k} \mathbf{S} f_{k j}\right] \mathbf{W}^{i j} .
\end{gathered}
$$

The amplitude updates are then transformed back into the PAO basis,

$$
\begin{gathered}
\Delta \mathbf{t}_{\mathrm{PAO}}^{i}=\Delta \overline{\mathbf{t}}^{i} \mathbf{W}^{i i^{\dagger}}, \\
\Delta \mathbf{T}_{\mathrm{PAO}}^{i j}=\mathbf{W}^{i j} \Delta \overline{\mathbf{T}}^{i j} \mathbf{W}^{i j^{\dagger}} .
\end{gathered}
$$

Typically, two microiterations per macroiteration are sufficient to improve and stabilize convergence. Convergence of the macroriterations can be further improved using the DIIS (direct inversion of the iterative subspace) procedure. Sometimes it is also useful to add constant shifts to the denominators in Eqs. (14) and (15).

The correlation energy is computed by using an approximate LCCSD Lagrangian, with the assumption that the multipliers are equal to the (contravariant) amplitudes (which holds exactly in the LMP2 case, where this expression corresponds
TABLE I. Comparison of CCSD and LCCSD convergence. The number of iterations needed to converge the amplitudes to an accuracy of $10^{-6}$ is shown. The convergence criterion is that the square norms of the singles and doubles amplitude changes are both smaller than $10^{-12}$. The aug-cc-pVTZ basis set was used.

\begin{tabular}{lccc}
\hline \hline Molecule & Canonical & Local $^{\mathrm{a}}$ & Local $^{\mathrm{b}}$ \\
\hline $\mathrm{C}_{2} \mathrm{H}_{2}$ & 11 & 11 & 10 \\
$\mathrm{C}_{4} \mathrm{H}_{4}$ & 10 & 11 & 10 \\
$\mathrm{CO}_{2}$ & 11 & 11 & 11 \\
$\mathrm{H}_{2} \mathrm{CO}$ & 12 & 13 & 11 \\
$\mathrm{H}_{2} \mathrm{O}_{2}$ & 13 & 14 & 12 \\
$\mathrm{C}_{2} \mathrm{H}_{5} \mathrm{OH}$ & 12 & 13 & 11 \\
$\mathrm{HCONH}_{2}$ & 13 & 14 & 13 \\
$\mathrm{HCOOH}$ & 13 & 13 & 12 \\
$\mathrm{HCOOCH}$ & 13 & 13 \\
\hline \hline
\end{tabular}

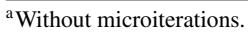

${ }^{\mathrm{b}}$ Using 2 microiterations per macroiteration.

to the Hylleraas functional),

$$
\begin{aligned}
E= & 2 \sum_{i} \sum_{r \in i} t_{r}^{i}\left(f_{r}^{i}+r_{r}^{i}\right)+\sum_{i j \in P_{s}} \sum_{r s \in[i j]} \tilde{D}_{r s}^{i j}\left(K_{r s}^{i j}+R_{r s}^{i j}\right) \\
& +\sum_{i j \in P_{w}} \sum_{r, s \in[i j]} \tilde{T}_{r s}^{i j} K_{r s}^{i j}
\end{aligned}
$$

with

$$
\begin{gathered}
f_{r}^{i}=\langle i|\hat{f}| r\rangle, \\
K_{r s}^{i j}=(i r \mid j s),
\end{gathered}
$$

representing the Fock matrix elements and two-electron integrals in the LMO/PAO basis, and

$$
D_{r s}^{i j}=T_{r s}^{i j}+t_{r}^{i} t_{s}^{j}, \quad i j \in P_{s},
$$

the composite LCCSD strong pair amplitudes. ${ }^{102}$ The weak pair amplitudes, $T_{r s}^{i j}$, ij $\in P_{w}$ are taken from a preceding LMP2 calculation. The tilde indicates contravariant quantities, i.e., $\tilde{T}_{r s}^{i j}=2 T_{r s}^{i j}-T_{s r}^{i j}$ and $\tilde{D}_{r s}^{i j}=2 D_{r s}^{i j}-D_{s r}^{i j}$. The singles and doubles residuals $r_{r}^{i}$ and $R_{r s}^{i j}$ go to zero at convergence. Their inclusion makes the energy expression approximately quadratic in errors of the amplitudes $t_{r}^{i}$ and $T_{r s}^{i j}$ and thus leads to faster convergence of the energy.

A comparison of the convergence of canonical and local coupled cluster calculations is presented in Table I. In the local case, calculations with and without the additional microiterations are compared. In the third column of the table, the off-diagonal elements $f_{i k}$ in Eqs. (16) and (17) are neglected, i.e., just the initial microiteration is performed. This corresponds to the usual update procedure in canonical calculations. In the last column, two microiterations per macroiteration were performed. Even though the improvements by using the microiterations are not dramatic, the number of iterations is never larger than for the canonical method. The microiterations also stabilize the convergence of the energy, and in no case more than nine iterations were needed to obtain an accuracy of $1 \mu \mathrm{H}$ for the LCCSD energy (in Table I more iterations were performed to accurately converge the amplitudes). 


\section{Choice of orbital domains}

In most previous applications of local correlation methods as outlined in Sec. II B, the orbital domains were determined by a procedure originally proposed by Boughton and Pulay (BP) (Ref. 68) (for details about domain specification in our program, see also Refs. 7 and 17). It turned out, however, that this choice is rather strongly basis set dependent. More recently, Mata and Werner ${ }^{18}$ proposed an alternative domain selection method that is based on natural population analysis ${ }^{69}$ (NPA) and much more stable with respect to the basis set. ${ }^{18}$ On the other hand, due to the lack of a clear localization criterion to be put into the Lagrangian the latter have the disadvantage of not being compatible with analytic energy gradient calculations. ${ }^{15,70}$

Both for the BP and NPA methods, the domains comprise all PAOs at a selected set of atoms and, therefore, an orbital domain is equivalent to a subset of atoms assigned to the LMO. The standard domains obtained by the BP or NPA procedure can be augmented by adding the PAOs at the next shell(s) of neighboring atoms. In this way, it is possible to approach the canonical result systematically. Typically, 98\%-99\% of the correlation energy of the corresponding canonical method for a given basis is recovered with standard domains; if the domains are extended by one shell of neighboring atoms, about $99.8 \%-99.9 \%$ of the full correlation energy is obtained. ${ }^{14,17}$ It should be noted, however, that domain extensions strongly increase the computational cost (for $\operatorname{LCCSD}(\mathrm{T})$, the computational resources such as CPU time and disk space increase with $L^{4}$, where $L$ is a measure of the average domain size per pair).

Finally we note that the domains may change as a function of the geometry. ${ }^{71-73}$ This can be avoided by freezing the domains determined at some geometry, e.g., the equilibrium distance. In most cases, this has very little effect on the results and guarantees microscopically smooth potentials. In case where the electronic structure strongly changes as a function of the geometry, it may be necessary to merge the domains obtained at different structures. This has been discussed and demonstrated in detail in Ref. 73.

\section{Pair approximations}

The second type of approximations in local correlation methods is to introduce a hierarchy of pair classes, which are treated at different levels. In our program, we distinguish strong, close, weak, distant, and very distant pairs. By default, only the strong pairs are included in the LCCSD, i.e., the summations over $i j$ are restricted as indicated in Eqs. (5) and (20). The close, weak, and distant pair amplitudes are optimized at the LMP2 level and kept fixed thereafter. For this purpose, an initial LMP2 calculation is performed for all (except very distant) pairs, and the pair energies for the close, weak, and distant pairs are added to the LCCSD energy in Eq. (20). One can also solve the LMP2 equations with fixed strong-pair amplitudes from the converged LCCSD, but we found that this makes virtually no difference for the accuracy of the results.

Very distant pairs are completely neglected. The integrals ( $r i \mid s j$ ) for distant pairs $i j$ can optionally be evaluated by multipole approximations, ${ }^{74,75}$ but this is mainly of interest to reduce the computational effort in LMP2 calculations for very large systems. For LCCSD, the multipole approximation would not save much time and is not recommended. Thus, in the current paper, there is no difference between weak and distant pairs.

Close pairs were originally introduced as a pair class intermediate between strong (LCCSD) and weak (LMP2) to specify restricted lists of triples for local (T) calculations ${ }^{10,11}$ (cf. Sec. II E). Furthermore, the close pair amplitudes, determined at the LMP2 level, are included in the triples calculation. Meanwhile they can also be included in the LCCSD residuals (option KEEPCLS=1). The inclusion of the close pair amplitudes in the LCCSD amplitude equations for the strong pairs requires only a relatively small additional effort. In particular, it does not affect the contributions of the 4-external integrals. A strong increase in the number of 3external integrals required in the LCCSD part can be avoided by neglecting the contributions that are quadratic in the amplitudes (cf. Appendix B). As will be shown in Ref. 1, the inclusion of close-pair amplitudes is particularly important in the DF-LCCSD(T)-F12 method, since the explicitly correlated terms remove most of the domain error and then the errors caused by the pair approximations dominate.

The assignment of orbital pairs to the pair classes depends on the distance $R_{i j}$ of the LMOs $\phi_{i}$ and $\phi_{j}$. This is defined as the minimum distance between any atom in the domain $[i]$ and any atom in the domain $[j]{ }^{7}$ Alternatively, one can use the minimum number of bonds between these two sets of atoms. The advantage of the latter method is that it is independent of the bond lengths and works for any molecule. The disadvantage is that under certain circumstances atoms which are separated by many bonds can be spatially close (an example will be presented at the end of Sec. IV). This problem can be avoided by using distance criteria to determine the distant and very distant pairs, and connectivity criteria for the remaining pair classes. Furthermore, it is sometimes useful to determine the pair lists at one geometry (along with the domains) and then to keep them fixed at other geometries, as already discussed for the domains in Sec. II C. For example, this is important when bonds are elongated at transition states. $^{76,77}$ This is also the preferred way to avoid basis set superposition errors (BSSE) in local correlation calculations on intermolecular complexes and clusters., 78,79

Unless otherwise noted, we will use connectivity criteria in the current paper, and these will be defined in Sec. IV.

\section{E. Triple excitations}

In order to compute the local perturbative triples correction (T), ${ }^{10,11}$ the triple excitations $\hat{E}_{i}^{r} \hat{E}_{j}^{s} \hat{E}_{k}^{t} T_{r s t}^{i j k}$ are restricted to triples domains $[i j k]=[i] \cup[j] \cup[k]$. Furthermore, the list of orbital triples $i j k$ is (by default) restricted such that the related pairs $i j, i k, k j$ are close or strong, and at least one of them is strong. The strong and close pair amplitudes as well as the singles amplitudes are included in the triples equations.

Since the Fock matrix is not diagonal in the local basis, the zeroth-order Hamiltonian is not diagonal in the basis of triply excited determinants, and the perturbative triples equations need in principle to be solved iteratively. Even though 
this is possible, ${ }^{11}$ it is expensive and requires to store all triples amplitudes. By neglecting couplings induced by offdiagonal elements $f_{i j}$ in the occupied-occupied block of the Fock matrix the equations can be decoupled (T0 approximation). Alternatively, the $f_{i j}$ couplings can be treated by first-order perturbation theory, which also avoids storage of the triples amplitudes, ${ }^{11}$ yet is considerably more expensive than the T0 approximation. Since the T0 triples correction can be computed very efficiently and in most cases provides sufficient accuracy it has been used throughout in the present work.

The computational effort scales strictly linear with molecular size. Thus, in sharp contrast to canonical methods, where calculation of the (T) correction is the main bottleneck, the evaluation of the local (T0) corrections usually takes only a comparable or even shorter time than the LCCSD. It should be noted, however, that the relative effort for the triples quite strongly increases if the close-pair class is extended.

\section{F. Further approximations to achieve linear scaling}

The domain approximation in combination with the restriction of the LCCSD treatment to strong pairs automatically leads to linear scaling for most terms in the LCCSD equations. This is due to the fact that the residuals $R_{r s}^{i j}$ have to be computed only for the domains $r, s \in[i j]$ and that the occupied pair labels of the residuals and amplitude matrices are restricted to strong (or close) pairs. However, there are some terms, in particular those involving single excitations, for which this alone is not sufficient to achieve linear scaling. One also has to exploit that certain integrals decay quickly with the distance between the orbitals involved. This leads to the definition of integral lists and integral domains as discussed in detail in Ref. 9. These lists and domains can be determined in advance on the basis of distance criteria, which makes it possible to achieve linear scaling not only in the computation of the residuals, but also in the integral transformations. The errors introduced by these truncations only amount to a few microhartree and are negligible. We note that in the current work some additional approximations have been introduced to the contributions of the 3-external integrals in the pair-singles coupling terms (cf. Appendix B for details). These strongly reduce the number of 3-external integrals to be computed and stored.

The only term for which linear scaling is not easily achieved ${ }^{9}$ involves the contraction,

$$
[\mathbf{G}(\mathbf{E})]_{r s}=\sum_{i}[2(r s \mid i u)-(r i \mid s u)] t_{u}^{i} .
$$

This matrix is needed for all $r, s$ since it contributes to all pairs [cf. Eqs. (B21) and (B24) in Appendix B]. Calculating $\mathbf{G}(\mathbf{E})$ can entirely be avoided by using an approximation which is based on the observation that these terms cancel to large extent with the corresponding terms involving 1-external, instead of the 3-external integrals, i.e., with the terms involving $\mathbf{l}^{k l i}$ in the $\beta_{i k} \mathbf{S T}^{k j} \mathbf{S}$ contribution to the residuals $\mathbf{R}^{i j}$ [cf. Eqs. (B21) and (B23) in Appendix B]. If these contributions along with those of $\mathbf{G}(\mathbf{E})$ are neglected (option SKIPGE $=1$ ), only a small error results. ${ }^{9}$ This error is much smaller than errors caused by the domain approximation, and it is also much smaller than the difference between full CCSD and quadratic configuration interaction. In the latter method, these terms are also absent. The effect of this approximation on the accuracy and efficiency will be demonstrated in Sec. IV.

\section{LOCAL DENSITY FITTING APPROXIMATIONS}

\section{A. 0-2 external integrals}

The 2-external exchange integrals $K_{r s}^{k l}=(r k \mid l s)(r s$ $\left.\in[k l]_{\mathrm{K}}\right)$ are computed using density fitting as described in detail in Ref. 14. The all-internal and 1-external integrals can be obtained in the same way with very little extra cost. Since for LCCSD the operator lists $k l$ and operator domains $[k l]_{\mathrm{K}}$ are much larger than in the LMP2 case, ${ }^{9}$ it is usually not useful to enable local fitting - the crossover point to linear scaling occurs quite late, and for medium size calculations the overhead exceeds the savings. However, despite the fact that without local fitting the algorithm scales in the asymptotic limit cubically (with a low prefactor), the CPU times for this part of the transformation are quite short and have never been a bottleneck in the calculations.

The 2-external Coulomb integrals $J_{r s}^{i j}=(r s \mid i j)$ are first computed in the $\mathrm{AO}$ basis as

$$
(\mu \nu \mid i j)=(\mu \nu \mid A) d_{i j}^{A}
$$

where $d_{i j}^{A}$ are the fitting coefficients obtained in the generation of the 0 -external integrals, i.e.,

$$
\sum_{B}(A \mid B) d_{i j}^{B}=(A \mid i j) .
$$

Here and in the following, $A, B$ refer to fitting basis functions $\chi_{A}, \chi_{B}$, and the 2-index and 3-index Coulomb integrals are defined as

$$
\begin{gathered}
(A \mid B)=\int \chi_{A}\left(\mathbf{r}_{1}\right) r_{12}^{-1} \chi_{B}\left(\mathbf{r}_{2}\right) d \mathbf{r}_{1} d \mathbf{r}_{2}, \\
(\mu \nu \mid A)=\int \chi_{\mu}\left(\mathbf{r}_{1}\right) \chi_{\nu}\left(\mathbf{r}_{1}\right) r_{12}^{-1} \chi_{A}\left(\mathbf{r}_{2}\right) d \mathbf{r}_{1} d \mathbf{r}_{2} .
\end{gathered}
$$

The fitting coefficients $d_{i j}^{A}$ are kept in memory. The outermost loop runs over blocks of AOs, $\mu \geq v$, and for each block the three-index integrals are computed and contracted on the fly with the fitting coefficients. In the final step the integrals are transformed into the PAO basis. In the asymptotic limit, the algorithm scales cubically with molecular size, but as for the 2-external exchange integrals the time is usually short as compared to other parts of the calculation.

\section{B. 3- and 4-external integrals}

The 3-external integrals $(r s \mid k t)$ occur in various terms involving contractions with the singles amplitudes,

$$
\begin{aligned}
{\left[\mathbf{J}\left(\mathbf{E}^{k j}\right)\right]_{r s} } & =(r s \mid k t) t_{t}^{j}, \\
{\left[\mathbf{K}\left(\mathbf{E}^{k j}\right)\right]_{r s} } & =(r k \mid s t) t_{t}^{j},
\end{aligned}
$$


and with doubles amplitudes,

$$
\left[\mathbf{K}\left(\mathbf{D}^{i j}\right)\right]_{r k}=(r s \mid k t) D_{s t}^{i j},
$$

as well as in the expression of the perturbative triples correction (see Appendix B for the explicit LCCSD equations). The range of indices $r, s, t$ for each LMO $k$ is independent of the molecular size and can be determined in advance (3-ext domains). Thus, in the asymptotic limit, the number of these integrals scales linearly with molecular size. A detailed description of the definition of the 3-ext domains can be found in Appendix B.

The 4-external integrals $(r t \mid s u)$, on the other hand, just appear in the external exchange operators,

$$
\left[\mathbf{K}\left(\mathbf{D}^{i j}\right)\right]_{r s}=(r t \mid s u) D_{t u}^{i j}, \quad r, s, t, u \in[i j],
$$

which contribute only to the doubles residual for pair $i j$. Since all four indices of $(r t \mid s u)$ always belong to the same domain, this set of integrals is usually as compact as the 3-external integral set, in spite of the additional virtual orbital index. Obviously, since the number of correlated pairs $i j$ scales linearly with molecular size, and the pair domains are independent of the molecular size, also the number of 4-external integrals scales linearly. However, different pair domains may overlap and, therefore, the calculation and storage of a different integral set for each domain $[i j]$ would be highly redundant. A non-redundant set of 4-external integrals can be determined by first setting up a non-redundant list of center quadruples $(R S T U)$; this makes use of the fact that the domains always include all PAOs at a given center. The calculation and contraction of the 4-external integrals are then driven by this list. ${ }^{13}$ Quite similarly, the 3-external integral set is driven by a quadruple ( $k R S T)$ consisting of one LMO index $k$ and three centers, specifying the minimum set of integrals required for the computation of all the terms as mentioned above.

As the 0-2 external integrals, the 3- and 4-external integrals are generated by utilizing density fitting. For the sake of simplicity, each of these three subsets is computed independently. In principle, common intermediates such as the transformed 3-index integrals $(r i \mid A)$ and $(r s \mid A)$ and the corresponding fitting coefficients could be computed only once and reused, but this has not been implemented. The main reason for this is that the domain sizes for the individual integral subsets are different, and this would cause considerable complications.

Since the individual orbital product distributions are local (products of spatially local one-electron functions), local fitting can be applied. In the following, we outline our algorithm for the 4-external integrals, for which local fitting is most useful. Local fitting for the 3-external integrals proceeds along similar lines. However, since the 3 -external domains are quite extended, the savings are smaller than in the 4-external case, and the crossover point to the standard non-local fitting method occurs only for larger molecular sizes. An example where local fitting indeed leads to substantial savings is given in Sec. IV (Table IX).
Local fitting makes it necessary to use the robust threeterm fitting expression, ${ }^{14,16,80-82}$

$$
\begin{aligned}
(r s \mid t u) \approx & \sum_{A \in[R S]} d_{r s}^{A}(A \mid t u)+\sum_{B \in[T U]} d_{t u}^{B}(B \mid r s) \\
& -\sum_{A \in[R S]} \sum_{B \in[T U]} d_{r s}^{A}(A \mid B) d_{t u}^{B}
\end{aligned}
$$

or the symmetric two-term term formula, ${ }^{16}$

$$
(r s \mid t u) \approx \frac{1}{2}\left[\sum_{A \in[R S]} d_{r s}^{A}(A \mid t u)+\sum_{B \in[T U]} d_{t u}^{B}(B \mid r s)\right] .
$$

Here, $A \in[R S]$ represents an auxiliary fitting function confined to a fit domain $[R S]$. This domain is related to the charge distributions of the orbital products $\phi_{r}^{\mathrm{PAO}}(\mathbf{r}) \phi_{s}^{\mathrm{PAO}}(\mathbf{r})$, where $r$ and $s$ denote functions located at the centers $R$ and $S$, respectively. Thus, the fitting domains are specific to center pairs. $d_{r s}^{A}$ are the fitting coefficients obtained by solving for each center pair $R S$,

$$
\sum_{B \in[R S]}(A \mid B) d_{r s}^{B}=(A \mid r s), \quad \forall A \in[R S] .
$$

Since this implies that the indices $A, B, r, s$ refer to functions that are spatially close, the computational effort scales linearly with molecular size. In the case that full fit domains are used (non-local fitting), all terms in Eqs. (33) and (34) are identical, yielding the usual one-term expression of density fitting.

In Ref. 16, a first implementation of local fitting for the 4-external integrals was presented. There the individual fit domains $[R S]$ were comprising the fitting functions of the centers of all center quadruples (RSTU) containing the center pair $(R S)$. Optionally, further centers could be added to these core fit domains based on a distance criterion. Such an approach, however, can only efficiently work if the correlated orbitals $i$ and $j$ are spatially close. This is because the pair domains $[i j]$ are the union of the orbital domains $[i]$ and $[j]$ and, therefore, the size of the fitting domain would depend on the number of orbitals $j$ that form pairs with a given orbital $i$. In the case of extended pair lists or domains, this scheme becomes inefficient and a more advanced algorithm is desirable. In the present work, we adopt a procedure developed for density fitting in the context of periodic systems, ${ }^{57,58,83,84}$ where local fitting is not only more efficient, but also mandatory due to the infinite nature of the physical system. The importance of a certain center $X$ for the product charge distribution of orbitals $r, s$ is measured by the quantity, ${ }^{58}$

$$
q_{r s}^{X}=\left(1+\tau_{r s}\right) \sum_{\mu \in X}\left(\sum_{\nu \in X} P_{\mu r} S_{\mu \nu} P_{\nu s}\right)^{2},
$$

where $P_{\mu r}$ are the PAO coefficients as defined in Eq. (3) and $\tau_{r s}$ permutes the indices $r$ and $s$. This "pseudo population" then is condensed to individual groups of product distributions for each center pair $R S$ (for product distributions 
$r s)$ as

$$
q_{R S}^{X}=\sum_{r \in R} \sum_{s \in S} q_{r s}^{X} .
$$

The quantities $q_{R S}^{X}$ are finally normalized over the center range $X$ and sorted for each individual center pair $(R S)$. Core fit domains then are specified for $R S$ by adding more and more centers $X$ from the sorted list, until a certain threshold is reached. The core fit domains can optionally be augmented by further centers according to either a distance or a topology criterion. The set of three-index integrals $(A \mid t u)$ required in the assembly step of the 4-external integrals adheres to united fit domains,

$$
(t u \mid A), \quad A \in[R S], \forall(R S) \in(R S T U) .
$$

\section{SAMPLE CALCULATIONS}

The method presented in Secs. I-III is available in the MOLPRO package of ab initio programs ${ }^{85,86}$ and has already been successfully applied in various applications. ${ }^{19,76,77,87-90}$ For example, barrier heights in various enzymes have been computed in the framework of the hybrid quantum mechanical and molecular mechanics (QM/MM) approach, and the effect of domain and pair approximations on the results has been systematically investigated. ${ }^{76,77,90}$ Furthermore, the accuracy of the local coupled cluster method (without density fitting) has been tested for reaction energies ${ }^{17}$ and other molecular properties, such as dipole moments and dipole polarizabilities. ${ }^{89}$ Especially noteworthy is the possibility to correlate only certain regions of a molecule in the vicinity of the reaction center. ${ }^{19}$ Then the computation time for the correlation calculation becomes independent of the size of the rest of the molecule. It has been shown in Ref. 19 that rela- tively small regions are sufficient to obtain converged reaction energies.

Here, we focus on the accuracy and efficiency of the density fitting, as well as of pair approximations. The test molecules considered for that purpose are shown in Fig. 1. The geometries of these molecules were optimized by DFLMP2 using the cc-pVTZ basis set. ${ }^{91}$ We have studied three reactions involving these molecules, namely, the hydrogenation of progesteron via pregnenolon to pregnanediol (reactions I and II), and the last synthetic step to yield androstendione (reaction III),

$$
\begin{aligned}
& \text { Reaction I : } \mathrm{C}_{21} \mathrm{O}_{2} \mathrm{H}_{30}+\mathrm{H}_{2} \rightarrow \mathrm{C}_{21} \mathrm{O}_{2} \mathrm{H}_{32} . \\
& \text { Reaction II : } \mathrm{C}_{21} \mathrm{O}_{2} \mathrm{H}_{32}+2 \mathrm{H}_{2} \rightarrow \mathrm{C}_{21} \mathrm{O}_{2} \mathrm{H}_{36} . \\
& \text { Reaction III : } \mathrm{C}_{25} \mathrm{O}_{4} \mathrm{H}_{32} \rightarrow \mathrm{C}_{19} \mathrm{O}_{2} \mathrm{H}_{26}+\mathrm{C}_{6} \mathrm{O}_{2} \mathrm{H}_{6} .
\end{aligned}
$$

Moreover, as an illustrative example for the capabilities of our program, we will present some preliminary results on the thermal reaction energy of the photodamaged cyclobutane pyrimidine dimer (CPD) lesion back to the undamaged/repaired dimer (TpT) in the ATTA sequence of the DNA double strand.$^{92}$ Further benchmarks, comparing the standard DF-LCCSD(T) method with the explicitly correlated variant DF-LCCSD(T)-F12 are presented in Ref. 1.

As orbital basis we used the correlation consistent triple$\zeta$ basis sets, cc-pVTZ. ${ }^{93}$ Diffuse functions were added for the oxygen and nitrogen atoms, i.e., $\mathrm{C}, \mathrm{H}=\mathrm{cc}-\mathrm{pVTZ}, \mathrm{O}, \mathrm{N}=$ augcc-pVTZ. ${ }^{94}$ In the following, this mixed basis will be denoted by aVTZ. In order to study basis set effects, the corresponding aVQZ and aV5Z basis sets were used as well. The auxiliary basis sets for the Fock-matrix fitting ${ }^{46}$ in the preceding density fitted Hartree-Fock (DF-HF) calculations were cc-pVnZ/JKFIT (Ref. 45) ( $n=\mathrm{T}, \mathrm{Q}, 5$ corresponding to the OBS). For all other integrals, the cc-pVTZ/MP2FIT,

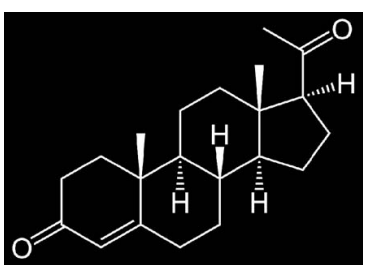

(a) Progesteron

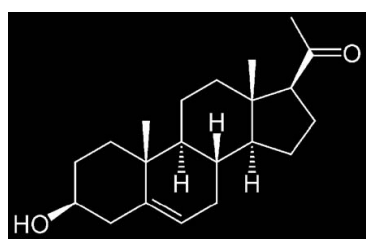

(b) Pregnenolon

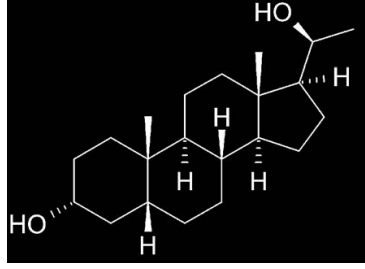

(c) Pregnanediol

(d)

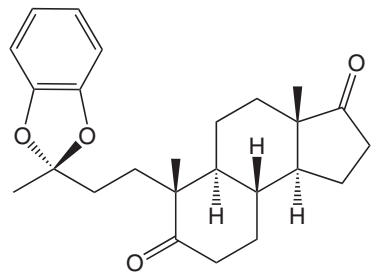

Androstendione Precursor

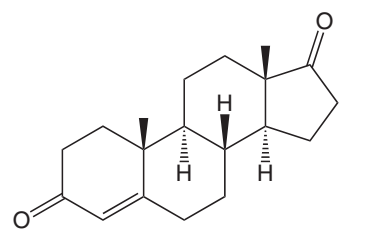

Androstendione<smiles>Oc1ccccc1O</smiles>

Orthohydroxyphenol

FIG. 1. The molecules (a) progesterone $\mathrm{C}_{21} \mathrm{O}_{2} \mathrm{H}_{30}$, (b) pregnenolone $\mathrm{C}_{21} \mathrm{O}_{2} \mathrm{H}_{32}$, (c) pregnanediol $\mathrm{C}_{21} \mathrm{O}_{2} \mathrm{H}_{36}$, and (d) a reaction step yielding androstendione. 
TABLE II. Definition of pair classes based on connectivity criteria. Here, $n$ is the number of bonds between the orbital domains $[i]$ and $[j]$ that form a pair $i j$. The input parameters IVDIST, IWEAK, ICLOSE, and KEEPCLS are abbreviated as $v, w, c$, and $k$, respectively. In the current work, there is no difference between weak and distant pairs. Unless otherwise noted the default IVDIST $=8$ was used.

\begin{tabular}{lll}
\hline \hline Pair class & \multicolumn{1}{c}{ Criteria } & \multicolumn{1}{c}{ Amplitudes } \\
\hline Strong & $n<c$ & Optimized by LCCSD \\
Close $^{\mathrm{a}}$ & $c \leq n<w$ & $\begin{array}{l}\text { Optimized by LMP2 } \\
\text { but included in LCCSD(T0) }\end{array}$ \\
Weak & $w \leq n<v$ & Optimized by LMP2, \\
& & neglected in LCCSD(T0) \\
Very distant & $v \leq n$ & Entirely neglected \\
\hline \hline
\end{tabular}

a If $k=0$, these pairs are neglected in the LCCSD residuals but included in the (T0) calculation. If $k=1$, they also contribute to the strong pair and singles LCCSD residuals.

cc-pVQZ/MP2FIT, or cc-pV5Z/MP2FIT (Ref. 50) basis sets have been used; as for the OBS, the corresponding augmented basis sets were used for the oxygen and nitrogen atoms. Natural localized molecular orbitals were employed, and unless otherwise noted the NPA with the threshold NPASEL $=0.07$ was used to determine the domains as described in Ref. 18. Option MERGEDOM=1 was used, which merges overlapping domains of the $\pi$-orbitals in aromatic rings of reaction III, yielding identical domains that extend over all six atoms (for all other reactions studied in this paper there is no effect). Local fitting was used only when indicated.

As outlined in Sec. II D, the definition of the pair classes is based on the number of bonds $(n)$ between the closest atoms of the two orbital domains of a pair. In the current benchmarks, the numbers of strong, close, and weak pairs are varied. The parameters IWEAK and ICLOSE affect the assignment of the pairs to these three classes. The additional parameter KEEPCLS affects the approximations made for the different pair classes. The meaning of the three parameters is summarized in Table II. In the following, we will use the abbreviation $w c k$, so that, e.g., $w c k=321$ means $\operatorname{IWEAK}=3$, $I C L O S E=2, K E E P C L S=1$. In addition, we will test the effect of neglecting $\mathbf{G}(\mathbf{E})$ along with the corresponding contributions of the one-external integrals (option $\mathrm{SKIPGE}=1$, cf. Sec. II F).

We will first test the errors caused by the density fitting approximation. Table III shows the errors of the DF-

TABLE III. Fitting errors $\Delta$ and additional local fitting errors $\delta_{\text {symm }}$ (using symmetric formula, Eq. (34)), $\delta_{\text {robust }}$ (using robust formula, Eq. (33)) for pregnanediole, DF-LCCSD/aVTZ. All values are given in $\mu E_{h}$. For the LCCSD method, the errors refer to the total correlation energy (including the weak pair LMP2 contributions) for (T0) to the triples contribution only.

\begin{tabular}{lrrrrr}
\hline \hline $\begin{array}{l}\text { 0-3 ext. } \\
\text { 4-ext. }\end{array}$ & $\begin{array}{r}\text { aVTZ } \\
\text { aVTZ }\end{array}$ & $\begin{array}{r}\text { aVTZ } \\
\text { aVQZ }\end{array}$ & $\begin{array}{r}\text { aVQZ } \\
\text { aVQZ }\end{array}$ & $\begin{array}{r}\text { aVQZ } \\
\text { aV5Z }\end{array}$ & $\begin{array}{r}\text { aV5Z } \\
\text { aV5Z }\end{array}$ \\
\hline$\Delta$ (LMP2) & 458 & 458 & 157 & 157 & 5 \\
$\Delta$ (LCCSD) & -1976 & 147 & -100 & 103 & -22 \\
$\delta_{\text {symm }}($ LCCSD) & -150 & -43 & -39 & -12 & -10 \\
$\delta_{\text {robust }}($ LCCSD) & -151 & -42 & -39 & -10 & -9 \\
$\Delta($ T0) & -78 & -71 & -3 & -3 & -1 \\
$\delta_{\text {symm }}($ T0 $)$ & -22 & -20 & 0 & 0 & 1 \\
$\delta_{\text {robust }}($ T0 $)$ & -8 & -5 & 0 & 0 & 0 \\
\hline \hline
\end{tabular}

TABLE IV. Effect of density fitting approximations on reaction energies (in $\mathrm{kJ} \mathrm{mol}^{-1}$ ). $w c k=210$, SKIPGE $=0$. Basis: aVTZ

\begin{tabular}{|c|c|c|c|c|c|c|c|}
\hline \multicolumn{2}{|c|}{ Fitting basis } & \multicolumn{2}{|c|}{$\mathrm{HF}$} & \multicolumn{2}{|c|}{ LCCSD } & \multicolumn{2}{|c|}{ LCCSD(T0) } \\
\hline $0-3$ ext. & 4-ext. & I & II & I & II & I & II \\
\hline \multicolumn{2}{|c|}{ Integral-direct } & -59.2 & -195.0 & -75.0 & -218.6 & -70.9 & -209.4 \\
\hline aVTZ & aVTZ & -59.3 & -195.1 & -75.2 & -218.7 & -71.1 & -209.5 \\
\hline aVTZ & aVQZ & -59.3 & -195.1 & -75.2 & -218.8 & -71.1 & -209.6 \\
\hline
\end{tabular}

LCCSD correlation energy of pregnanediol as compared to an integral-direct LCCSD calculation without density fitting. For comparison, the errors of the corresponding DF-LMP2 calculations are also presented. It is found that fitting the 0-3 external integrals in the DF-LCCSD does not lead to an error that is significantly larger than for DF-LMP2, even though the fitting basis set was optimized for MP2 and not for LCCSD. However, the fitting approximation is more critical for the 4external integrals, since these involve more virtual orbitals with a complicated node structure. This leads to an error that is 4-5 times larger (and of opposite sign) than the fitting error in DF-LMP2. The error of the correlation energy is reduced by one order of magnitude if for the 4-external the larger aVQZ/MP2FIT basis is used. It is then actually smaller than the DF-LMP2 error, but this is certainly due to some error compensations between the different integral classes. The errors due to local fitting are one order of magnitude smaller than the fitting errors and thus negligible.

Table IV demonstrates the effect of the density fitting on reaction energies. The Hartree-Fock values are affected by $0.1 \mathrm{~kJ} \mathrm{~mol}^{-1}$, the LCCSD, and LCCSD(T0) correlation contributions by about the same amount. These errors are certainly negligible, even if only the aVTZ fitting basis is used for the 4-external integrals. In all subsequent calculations, the aVTZ/MP2FIT fitting basis set thus is used for all integral classes.

The savings due to the density fitting approximations are enormous. For pregnanediol, the standard integral-direct calculation of the transformed integrals [using the programs described in (Refs. 9 and 95)] is about 50 times slower than with the current density fitting implementation. This ratio would further increase if larger basis sets were used.

TABLE V. CPU times (in min on Intel W5590 @ 3.33 GHZ) for density fitting integral transformations for pregnanediol. Basis set: aVTZ.

\begin{tabular}{lccc}
\hline \hline Integral set & $w c k$ & locfit $=0$ & locfit=1 \\
\hline 0-2-external & 210 & 20.9 & 32.7 \\
& 311 & 43.8 & 47.0 \\
3-external & 321 & 44.7 & 47.5 \\
& 210 & 53.3 & 33.6 \\
& 311 & 76.1 & 50.3 \\
4-external & 321 & 113.7 & 81.1 \\
& 210 & 46.6 & 23.7 \\
& 311 & 38.1 & 19.2 \\
\hline \hline
\end{tabular}


TABLE VI. Canonical DF-HF and DF-MP2 results for reaction energies (see text).

\begin{tabular}{lccccccc}
\hline \hline & \multicolumn{3}{c}{ DF-HF } & & \multicolumn{3}{c}{ DF-MP2 } \\
\cline { 2 - 4 } \cline { 6 - 8 } BASIS & I & II & III & I & II & III \\
\hline aVTZ & -59.3 & -195.1 & -2.7 & -73.2 & -220.6 & 28.3 \\
aVQZ & -58.6 & -192.9 & -5.1 & -74.5 & -220.4 & 22.3 \\
aV5Z & -58.6 & -192.7 & -5.8 & -74.7 & -219.7 & 20.5 \\
CBS[45] & -58.6 & -192.6 & -5.9 & -74.9 & -219.4 & 19.2 \\
\hline \hline
\end{tabular}

Table V shows the CPU times to generate the transformed integrals for pregnanediol with and without local density fitting. As already mentioned, there is no saving for the $0-2$ external integrals - in contrary, the overhead for local fitting increases the CPU times. Therefore, local fitting is not recommended for this class of integrals. However, there are significant savings for the 3-external and 4-external integrals. The time to generate the latter is almost reduced by a factor of 2 . Naturally, the savings get somewhat smaller if the pair lists are extended, since then the linear scaling regime is reached later.

Next we will assess the domain errors and basis set effects on the reaction energies. Normally, canonical DF-MP2 calculations are easily possible for molecules that can be treated by DF-LCCSD(T0). This makes it possible to estimate the domain error by comparison of DF-LMP2 and DFMP2 calculations. Experience for smaller molecules, where canonical coupled cluster calculations are still possible, has shown that the domain errors are very similar at the LMP2 and LCCSD levels, and thus it is possible to add a correction $\Delta E_{\mathrm{MP} 2}=E_{\mathrm{MP} 2}-E_{\mathrm{LMP} 2}$ to the LCCSD(T0) results. If DFMP2 results are available for larger basis sets, this may also include a basis set correction. The DF-MP2 reaction energies for aVTZ, aVQZ, and aV5Z, as well as the extrapolated basis set limits are presented in Table VI. The latter have been obtained by Karton-Martin extrapolation ${ }^{96}$ of the Hartree-Fock values and by $n^{-3}$ extrapolation ${ }^{97}$ of the correlation energies. The corresponding DF-LMP2/aVTZ values for reactions IIII are $-69.2,-211.5$, and $15.3 \mathrm{~kJ} \mathrm{~mol}^{-1}$, respectively, yielding domain corrections of $-4.0,-9.1$, and $+13.0 \mathrm{~kJ} \mathrm{~mol}^{-1}$, respectively. The additional basis set corrections are -1.7 , +1.2 , and $-9.1 \mathrm{~kJ} \mathrm{~mol}^{-1}$, yielding total corrections of -5.7 , -7.9 , and $+3.9 \mathrm{~kJ} \mathrm{~mol}^{-1}$.

In particular for reaction III the domain error of $13 \mathrm{~kJ} \mathrm{~mol}^{-1}$ is quite large. It can be observed, however, that it has opposite sign as the basis set error, and actually the LMP2/aVTZ value is in much better agreement with the MP2/CBS value than with the MP2/aVTZ one. We believe that this is due to strong BSSE effects in the canonical calculation. It is well known that BSSE effects on the correlation energies are strongly reduced by the local ansatz. 7,78,79 In molecular clusters, this is due to the fact that double excitations from one monomer to the other are excluded by the local ansatz. Similarly, the absence of double excitations from one part of the molecule to another can reduce intramolecular BSSE effects, though this is more difficult to quantify. In the current case, BSSE effects should favor the energy of the large precursor relative to the products, making the
TABLE VII. Effect of weak pair approximations on correlation energies (in $E_{h}$ ), file sizes (in GB), and CPU times (in min on Intel Xeon W5590@ 3.33 GHZ) for pregnanediol. The Hartree-Fock energy is $-966.2468036 E_{h}$. The number of LCCSD iterations is given in parenthesis. Basis: aVTZ.

\begin{tabular}{|c|c|c|c|c|c|c|}
\hline \multirow[b]{2}{*}{$w c k$} & \multicolumn{2}{|c|}{ Correlation energy } & \multicolumn{2}{|c|}{ File size } & \multirow{2}{*}{$\begin{array}{l}\mathrm{CPU} \\
\text { time }\end{array}$} & \multirow[b]{2}{*}{ (it) } \\
\hline & LCCSD & $\operatorname{LCCSD}(\mathrm{T} 0)$ & 3 -ext & 4-ext & & \\
\hline 210 & -4.276074 & -4.433139 & 5.9 & 7.3 & 178.2 & $(8)$ \\
\hline $210^{\mathrm{a}}$ & -4.276081 & -4.433140 & 5.9 & 7.3 & 152.8 & (9) \\
\hline 211 & -4.240449 & -4.393467 & 6.2 & 7.3 & 188.6 & (9) \\
\hline 311 & -4.226976 & -4.394841 & 18.9 & 7.3 & 268.7 & (9) \\
\hline 411 & -4.222332 & -4.394565 & 37.8 & 7.3 & 380.2 & (10) \\
\hline 321 & -4.210514 & -4.377468 & 38.1 & 36.9 & 419.1 & (10) \\
\hline 421 & -4.205630 & -4.378183 & 48.5 & 36.9 & 611.7 & (10) \\
\hline 431 & -4.199469 & -4.371195 & 95.6 & 137.6 & 917.4 & (9) \\
\hline
\end{tabular}

${ }^{\mathrm{a}}$ Using SKIPGE $=1$. All other cases with SKIPGE $=0$.

MP2/aVTZ reaction energy too positive. Likely, this leads to a significant part of the observed difference of the LMP2/aVTZ and MP2/aVTZ values. This conclusion is supported by the fact that the LMP2/aVTZ reaction energy is in much better agreement with the MP2/CBS value than the MP2/aVTZ result. Thus, looking at the "domain error" for a fixed basis set may be misleading. Similar BSSE effects have, for example, also been found in calculations of vibrational frequencies, where the local results were found to be more accurate than the canonical ones. ${ }^{87,88}$ In order to account for all effects appropriately, we recommend to add a correction $\Delta E_{\mathrm{MP} 2(\mathrm{CBS})}=E_{\mathrm{MP} 2(\mathrm{CBS})}-E_{\mathrm{LMP} 2}$ to the LCCSD(T0) results. Alternatively, in Ref. 1 it will be shown that the basis set effect as well as the domain error can be accounted for more rigorously using explicitly correlated wavefunctions, and the final DF-LCCSD(T0)-F12 results are indeed close to the DF$\mathrm{LCCSD}(\mathrm{T} 0)+\Delta E_{\mathrm{MP} 2(\mathrm{CBS})}$ ones.

Next we will investigate the effect of the pair approximations. Table VII demonstrates the effect of the weak pair approximations on the correlation energies, file sizes, and CPU times for pregnanediole. A LCCSD(T0) calculation for this molecule with 59 atoms, 1226 contracted Gaussian basis functions (CGTOs), and the default parameters $w c k=210$ takes just $3 \mathrm{~h}$ of CPU time on a single core of an Intel W5590@3.33 GHZ processor. This can be reduced further to less than 2 h by neglecting the $\mathbf{G}(\mathbf{E})$ contributions (option SKIPGE =1, cf. Table VII) and by using local fitting for generating the 3-external and 4-external integrals (cf. Table V). Comparison with the timings in Table $\mathrm{V}$ shows that a large fraction of the total time is spent in the integral evaluation and transformation. The elapsed time depends sensitively on the amount of available memory and the I/O configuration; on our machine, which has $72 \mathrm{~GB}$ of memory and a striped file system with an I/O bandwidth of $500-600 \mathrm{MB} / \mathrm{s}$, the elapsed time for the smaller calculations $(w c k=210$ or 211$)$ is just 2\%-3\% larger than the user-CPU time. Most likely, in this case all integrals are cached in memory by the operating system. The situation is different, however, if the strong pair list is extended ( $w c k=321$ or larger); this increases the file sizes by a factor of more than 5 , and then the integrals cannot be cached in memory any more. The CPU time increases to about $7 \mathrm{~h}$, and the elapsed times are now about 
TABLE VIII. Effect of weak pair approximationson LCCSD(T0) reaction energies (in $\mathrm{kJ} \mathrm{mol}^{-1}$ ). Basis: aVTZ.

\begin{tabular}{lccccccc}
\hline \hline & \multicolumn{3}{c}{ LCCSD } & & \multicolumn{3}{c}{ LCCSD(T0) } \\
\cline { 2 - 4 } \cline { 6 - 8 }$w c k$ & I & II & III & I & II & III \\
\hline 210 & -75.0 & -218.5 & 22.2 & & -70.9 & -209.4 & 15.2 \\
$210^{\mathrm{a}}$ & -74.5 & -218.0 & 22.0 & -70.2 & -208.6 & 15.0 \\
211 & -74.9 & -219.2 & 23.3 & -71.3 & -211.0 & 16.8 \\
311 & -75.6 & -219.1 & 21.4 & -70.1 & -209.0 & 17.7 \\
411 & -75.5 & -218.6 & 18.8 & -69.9 & -208.6 & 16.9 \\
321 & -74.1 & -218.6 & 20.8 & -68.3 & -208.6 & 17.7 \\
421 & -73.9 & -217.8 & 18.0 & -68.1 & -207.9 & 17.0 \\
431 & -74.4 & -218.3 & 17.3 & -68.7 & -208.6 & 16.3 \\
\hline \hline
\end{tabular}

${ }^{\mathrm{a}}$ Using SKIPGE $=1$. All other cases with SKIPGE $=0$.

$20 \%$ larger than the CPU time. This is due to the fact that in the LCCSD iterations the processing of the 3-external and 4-external integrals is strongly I/O bound. It can be seen in Table VII that the correlation energies are reduced by including more pairs in the LCCSD residual. This is not due to an overestimation of the weak pair LMP2 energies, but due to the additional strong and weak pair couplings in the LCCSD. These couplings are approximately accounted for with option KEEPCLS=1, which means that the close-pair LMP2 amplitudes are included in the LCCSD residuals for the strong pairs. The standard choice of $w c k=210$ yields for pregnanediol a correlation energy that is about $1.4 \%$ larger than the best current value, obtained with $w c k=431$. This error is reduced to $0.5 \%$ by using $w c k=211$, i.e., KEEPCLS $=1$, and to $0.1 \%$ with $w c k=321$.

The LCCSD and LCCSD(T0) reaction energies are presented in Table VIII. The effect of SKIPGE $=1$ on the reaction energies is small $\left(0.2-0.8 \mathrm{~kJ} \mathrm{~mol}^{-1}\right.$ in the current examples). For reactions I and II, the LCCSD results are only weakly affected by the pair approximations. The variations depending on the choice of the $w c k$ parameter amount only to about $1 \mathrm{~kJ} \mathrm{~mol}^{-1}$. This is probably due to the fact that in these cases only $\mathrm{OH}$-bonds are formed and no additional long-range cor-

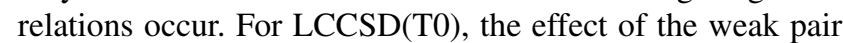
approximations on reactions I and II is somewhat larger than for LCCSD; overall, extending the strong pair list reduces the correlation effect on the reaction energies and, therefore, makes the reactions less exothermic. In this case, however, the choice $w c k=211$ yields somewhat worse agreement with the best values for the reaction energies, and only extending IWEAK to 3 improves the result. Apparently, different effects of opposite sign partly compensate each other.

For reaction III, the situation is different. Extension of the strong and/or close pair lists reduces the LCCSD reaction energy by about $5 \mathrm{~kJ} \mathrm{~mol}^{-1}$. Likely, this is due to the additional long-range correlation in the precursor relative to the separated molecules. This effect is to a large extent compensated, however, by the triples. Somewhat surprisingly, the effect of the triples on the reaction energy amounts to $-7 \mathrm{~kJ} \mathrm{~mol}^{-1}$ for $w c k=211$, but only $-1 \mathrm{~kJ} \mathrm{~mol}^{-1}$ for $w c k=431$. Thus, the $\operatorname{LCCSD}(\mathrm{T} 0)$ reaction energy is much less dependent on the choice of $w c k$ than the LCCSD one. At present, we have no simple explanation for this effect.
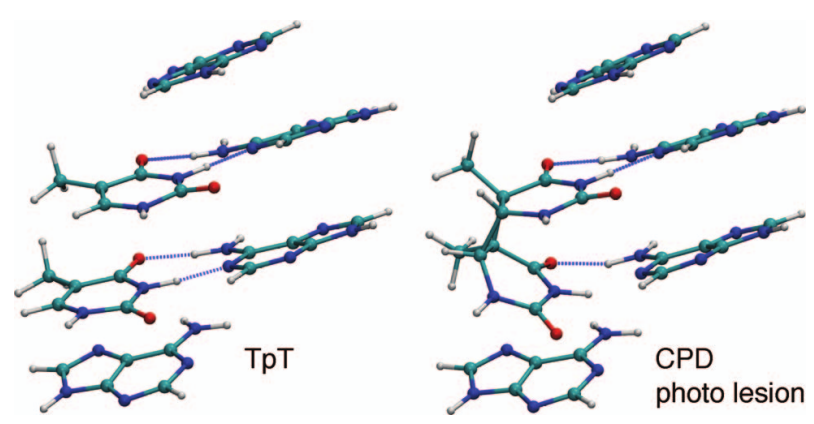

FIG. 2. Undamaged pyrimidine dimer (TpT) and the photoproduct cyclobutane pyrimidine dimer (CPD) thereof in the ATTA sequence of the DNA double strand. The geometry corresponds to frame D of Ref. 92 with the two pyrimidines H-bonded to the upper/lower adenines being removed. The chemical formulas of the two pyrimidines (thymines) and the four adenines are $\mathrm{C}_{5} \mathrm{H}_{6} \mathrm{~N}_{2} \mathrm{O}_{2}$ and $\mathrm{C}_{5} \mathrm{H}_{5} \mathrm{~N}_{5}$, respectively.

Furthermore, we point out that the domain and basis set corrections $\Delta E_{\mathrm{MP} 2(\mathrm{CBS})}$ to the $\mathrm{LCCSD}$ (T0) reaction energies yield estimates for the $\operatorname{CCSD}(\mathrm{T}) / \mathrm{CBS}$ values of $-74.4,-216.7$, and $20.2 \mathrm{~kJ} \mathrm{~mol}^{-1}$ for the three reactions, respectively. These compare very well with the $\operatorname{LCCSD}(\mathrm{T} 0)$ F12/aVTZ values of $-73.2,-215.9$, and $21.6 \mathrm{~kJ} \mathrm{~mol}^{-1}$, respectively. The latter values include the complementary auxiliary basis set (CABS) singles correction ${ }^{98,99}$ for basis set errors of the Hartree-Fock energies.

Finally, to demonstrate the efficiency of our program in the context of a small application project we present some preliminary results on the relative stability of the photodamaged CPD lesion vs. the undamaged dimer (TpT) in the ATTA sequence of the DNA double strand. ${ }^{92}$ The CPD is one important type of mutagenic photoproducts in DNA caused by solar irradiation in the UV spectral range, ${ }^{100}$ and the repair of these photolesions is of major importance for the survival of organisms. The CPD lesion is rather stable, and for its repair quite a large amount of energy is required to surmount the high reaction barriers. Nature, therefore, employs enzymes, such as photolyases, ${ }^{101}$ which exploit the energy of absorbed photons, to catalyse the repair reaction.

The present calculations comprise the CPD/TpT pyrimidine dimer plus the four nearest adenine bases (two hydrogen bonded, two $\pi$-stacked with respect to the pyrimidine dimer), in total 90 atoms, 528 correlated electrons, and 2636 basis functions in the aVTZ basis set. The geometries used for these calculations are depicted in Fig. 2 and correspond to frame D of Ref. 92. Since the intermolecular contribution to the relative stability of CPD vs. TpT is dominated by hydrogenbonding (the effect of $\pi$-stacking is small, cf. Fig. 4 in Ref. 92) the aVTZ basis as specified above was not further augmented by diffuse functions on the $\mathrm{C}$ atoms. For the local calculations NLMOs and NPA domains (NPASEL $=0.07$, vide supra) were used. In order to include intermolecular pairs, the individual pair classes were specified by employing distance rather than connectivity criteria, i.e., by $\left(r_{c}=1, r_{w}=5, r_{v d}=15\right.$ bohrs $)$. Pure connectivity criteria fail for systems comprising separate subunits or bent molecules. Close pairs were included in the computation of the LCCSD residuals (KEEPCLS $=1$, cf. Table II). The calculations were performed with SKIPGE $=0$, the $\mathbf{G}(\mathbf{E})$ terms 
TABLE IX. Relative energies $\Delta E=E(\mathrm{CPD})-E(\mathrm{TpT})\left(\right.$ in $\left.\mathrm{kJ} \mathrm{mol}^{-1}\right)$ and elapsed times (in $\mathrm{min}$ ) for various steps of the TpT calculation ( 7 cores, Intel Xeon X5690@3.47 GHz).

\begin{tabular}{|c|c|c|c|}
\hline \multirow[b]{2}{*}{ Method } & \multirow[b]{2}{*}{ Basis } & \multicolumn{2}{|c|}{$\Delta E$} \\
\hline & & locfit $=0$ & locfit $=1$ \\
\hline $\mathrm{HF}$ & aVTZ & 55.2 & \\
\hline $\mathrm{HF}$ & aVQZ & 55.4 & \\
\hline $\mathrm{HF}$ & $\mathrm{CBS}^{\mathrm{a}}$ & 55.4 & \\
\hline MP2 & $\mathrm{aVTZ}$ & 62.2 & \\
\hline MP2 & aVQZ & 61.0 & \\
\hline MP2 & $\mathrm{CBS}^{\mathrm{b}}$ & 60.1 & \\
\hline LMP2 & aVTZ & 66.3 & 66.2 \\
\hline LCCSD & aVTZ & 49.8 & 49.8 \\
\hline \multirow[t]{2}{*}{$\operatorname{LCCSD}(\mathrm{T} 0)$} & aVTZ & 61.5 & 61.7 \\
\hline & & \multicolumn{2}{|c|}{ Elapsed times } \\
\hline \multicolumn{4}{|l|}{ LMP2 } \\
\hline Integrals & & 45 & 18 \\
\hline Iterations & & 31 & 31 \\
\hline Total & & 80 & 50 \\
\hline \multicolumn{4}{|l|}{ LCCSD(T0) } \\
\hline 0-2-external & & 65 & 65 \\
\hline 3-external & & 166 & 87 \\
\hline 4-external & & 210 & 60 \\
\hline LMP2 It & & 31 & 31 \\
\hline LCCSD It & & 253 & 252 \\
\hline (T0) & & 62 & 63 \\
\hline Total & & 790 & 562 \\
\hline
\end{tabular}

${ }^{\mathrm{a}}$ Using Karton-Martin extrapolation.

${ }^{\mathrm{b}} \mathrm{Using} n^{-3}$ extrapolation of the correlation energies.

thus fully included. SKIPGE $=1$ calculations were tried but did not converge for the CPD system.

The resulting relative energies and the elapsed times of individual steps of the TpT calculation are compiled in Table IX. Evidently, a LCCSD(T0) calculation on a molecule of that size can be performed within less than half a day. The use of local fitting reduces the cost for integral generation considerably, for the 3-external, and even more for the 4-external integral set, with no effect on the accuracy of the final relative energies. Additional calculations employing the aVQZ and aV5Z fitting basis sets for the 4-external integrals showed that the relative energies remain entirely unaffected from an extension of the fitting basis.

Subtracting the local from the canonical MP2 value a domain error of $4.1 \mathrm{~kJ} \mathrm{~mol}^{-1}$ in the aVTZ relative energy is obtained. Using as correction term $\Delta E_{\mathrm{MP} 2(\mathrm{CBS})}=E_{\mathrm{MP2}(\mathrm{CBS})}$ $-E_{\mathrm{LMP} 2(\mathrm{aVTZ})}=6.2 \mathrm{~kJ} \mathrm{~mol}^{-1}$ yields an estimate for the corresponding $\mathrm{LCCSD}(\mathrm{T} 0)$ relative energy of $55.2 \mathrm{~kJ} \mathrm{~mol}^{-1}$, surprisingly close to the uncorrelated Hartree-Fock result. The DFT-SAPT calculations reported in Ref. 92 have shown that about $24 \mathrm{~kJ} \mathrm{~mol}^{-1}$ of the CPD vs. TpT relative stability comes from intermolecular interactions, i.e., the reduced strength of hydrogen bonding in the former, which apparently is a substantial fraction of the overall relative stability.

\section{SUMMARY AND CONCLUSIONS}

We have presented an efficient $\operatorname{LCCSD}(\mathrm{T})$ method and demonstrated that this makes it possible to study molecules with up to 90 atoms and over 2600 basis functions with a reasonable amount of computer time (over night). All integrals are computed using density fitting approximations. Depending on the basis set, this speeds up the calculations by 1-2 orders of magnitude, but has a negligible effect on absolute and relative energies. If local density fitting approximations are invoked, linear scaling of the computational effort can be reached, provided that certain terms involving contractions of single excitations are omitted.

For reactions involving such large molecules, the differences between the results of local and canonical calculations can be significant. This may either be due to the local approximations or the larger BSSE effects in the canonical calculations. The latter may either improve or deteriorate the canonical results relative to the basis set limit. Thus, there is often a strong coupling of the apparent domain and basis set effects which is difficult to predict. Therefore, it is recommended to add a correction $\Delta E_{\mathrm{MP} 2(\mathrm{CBS})}=E_{\mathrm{MP} 2(\mathrm{CBS})}-E_{\mathrm{LMP} 2}$ to the LCCSD(T0) energies, where the LMP2 and LCCSD(T0) energies are computed with the same basis set. Alternatively, these effects can be accounted for by using the explicitly correlated LCCSD(T0)-F12 method, which is presented in Ref. 1.

It has also been demonstrated that the errors caused by weak pair approximations may be larger than it was previously assumed. In some cases, the reaction energies may converge rather slowly with an extension of the strong and/or close pair classes. It is well known from previous work that in calculations with medium-size basis sets (e.g., cc-pVTZ) there is often a very favorable error compensation between the errors caused by the domain and pair approximations. ${ }^{17}$ However, the errors due to the pair approximation may become dominant if either the $\Delta E_{\mathrm{MP} 2 \text { (CBS) }}$ correction is added or the LCCSD(T0)-F12 method is used. Unfortunately, extending the number of strong and close pairs significantly increases the disk space and the CPU time. Nevertheless, near CCSD(T)/CBS accuracy can be reached for molecules with more than 100 atoms, such that high-level computational studies of many problems of current interest in (bio)chemistry and physics now become possible.

Extensions of the DF-LCCSD(T0) method presented here to open-shell cases ${ }^{24,25}$ are already functional and will be described in a future publication.

\section{ACKNOWLEDGMENTS}

This work was supported by the Deutsche Forschungsgemeinschaft (Leibniz program and SimTech Cluster of excellence at the University of Stuttgart).

\section{APPENDIX A: UNLINKED AND LINKED FORMS OF THE LCCSD RESIDUAL EQUATIONS}

In our previous LCCSD implementations, ${ }^{7,9}$ we defined the residuals as

$$
\begin{array}{cc}
\bar{r}_{r}^{i}=\left\langle\tilde{\Phi}_{i}^{r}|\hat{H}-E| \Psi\right\rangle & r \in[i], \\
\bar{R}_{r s}^{i j}=\left\langle\tilde{\Phi}_{i j}^{r s}|\hat{H}-E| \Psi\right\rangle & r, s \in[i j],
\end{array}
$$


i.e., according to the unlinked $\mathrm{CC}$ equations. Alternatively, one can use a similarity transformed Hamiltonian $\bar{H}$ $=e^{-\hat{T}} \hat{H} e^{\hat{T}}$ and write the residuals as

$$
\begin{gathered}
r_{r}^{i}=\left\langle\tilde{\Phi}_{i}^{r}\left|e^{-\hat{T}} \hat{H} e^{\hat{T}}\right| 0\right\rangle, \\
R_{r s}^{i j}=\left\langle\tilde{\Phi}_{i j}^{r s}\left|e^{-\hat{T}} \hat{H} e^{\hat{T}}\right| 0\right\rangle .
\end{gathered}
$$

These are the linked CC equations with each individual diagram contributing to the residuals being strictly size extensive by itself.

It can be shown that

$$
\begin{gathered}
\mathbf{r}^{i}=\overline{\mathbf{r}}^{i}, \\
\mathbf{R}^{i j}=\overline{\mathbf{R}}^{i j}-\mathbf{r}^{i} \mathbf{t}^{j^{\dagger}} \mathbf{S}-\mathbf{S t}^{i} \mathbf{r}^{j^{\dagger}} .
\end{gathered}
$$

In the canonical case (or if all domains comprise the full virtual space), the use of $\overline{\mathbf{R}}^{i j}$ leads to exactly the same results as with $\mathbf{R}^{i j}$, since for the optimized amplitudes the terms involving the singles residuals $\bar{r}_{r}^{i}$ vanish. We found that neither in terms of convergence nor in terms of computational efficiency, there is a particular advantage of using one or the other form. Yet in the local case the two different forms of the residual lead to slightly different results. The reason is that if single excitations are restricted to a domain of virtual orbitals $r \in[i]$, the residuals $r_{r}^{i}$ vanish only in this domain. Since for non-diagonal pairs $i j$ the pair domains $r, s \in[i j]$ are larger than the orbital domains $[i]$ or $[j]$, the subtracted terms in Eq. (A6) are non-zero even at convergence. In our current program Eq. (A4) is, therefore, used by default.

However, in our previous LCCSD implementations ${ }^{7,9}$ we used Eq. (A2). The doubles residuals then contained "unlinked" terms $s_{r}^{i} t_{s}^{j}$, where $s_{r}^{i}$ is a part of the singles residual and $r$ runs over the pair domain $[i j]$ (cf. Eqs. (42) and (44) in Ref. 7). Therefore, the vectors $s_{r}^{i}$ were computed for $r \in[i]_{\mathrm{U}}$, where $[i]_{\mathrm{U}}$ refers to the union of all pair domains $[i j]$ for a fixed $i$ (united pair domain). This caused additional complications and approximations in order to achieve linear scaling. If Eq. (A4) is used, the additional contributions are removed and all terms in the singles residual are only needed for $r \in[i]$. The current form [Eq. (A4)] is, therefore, preferable in local calculations. The results are hardly affected by this choice.

\section{APPENDIX B: THE LINKED LCCSD RESIDUALS}

In order to write the coupled cluster equations in compact and computationally efficient form, we define the contravariant doubles amplitudes, ${ }^{66,67}$

$$
\tilde{T}_{r s}^{i j}=2 T_{r s}^{i j}-T_{r s}^{j i} .
$$

The one- and two-electron integrals involving two external orbitals (PAOs) are ordered into matrices,

$$
\begin{gathered}
F_{r s}=\langle r|\hat{f}| s\rangle, \\
J_{r s}^{i j}=(r s \mid i j), \\
K_{r s}^{i j}=(r i \mid s j),
\end{gathered}
$$

and those with one external orbital into vectors,

$$
\begin{gathered}
f_{r}^{i}=\langle r|\hat{f}| i\rangle, \\
k_{r}^{k l i}=(r k \mid l i), \\
l_{r}^{k l i}=2(r k \mid l i)-(r l \mid k i) .
\end{gathered}
$$

The all-internal integrals are defined as

$$
\begin{gathered}
f_{i j}=\langle i|\hat{f}| j\rangle, \\
K_{k l}^{i j}=(i k \mid j l) .
\end{gathered}
$$

The superscripts denote different vectors or matrices, the subscript their elements. In the following matrices and vectors whose subscripts correspond to PAOs are typeset in upper and lower cases bold face letters, respectively, and the equations are given in matrix/vector form without specification of the external indices. The use of appropriate domains and pair restrictions is implied and not explicitly shown. Summation over repeated indices will always be implied.

The contributions of the 3-external and 4-external integrals only occur in contractions with amplitudes. Following the notation of earlier papers ${ }^{7,9,67}$ we define

$$
\begin{gathered}
{\left[\mathbf{K}\left(\mathbf{D}^{i j}\right)\right]_{r s}=(r t \mid s u) D_{t u}^{i j},} \\
{\left[\mathbf{K}\left(\mathbf{D}^{i j}\right)^{(k)}\right]_{r}=(r t \mid k u) D_{t u}^{i j},} \\
{\left[\mathbf{K}\left(\mathbf{E}^{i j}\right)\right]_{r s}=(r i \mid s u) t_{u}^{j},} \\
{\left[\mathbf{J}\left(\mathbf{E}^{i j}\right)\right]_{r s}=(r s \mid i u) t_{u}^{j},} \\
{[\mathbf{G}(\mathbf{E})]_{r s}=\sum_{i}\left[2 \mathbf{J}\left(\mathbf{E}^{i i}\right)-\mathbf{K}\left(\mathbf{E}^{i i}\right)\right]_{r s},}
\end{gathered}
$$

where formally $E_{p r}^{i j}=\delta_{p i} t_{r}^{j}$. For convenience in later expressions, we also define

$$
\begin{gathered}
\overline{\mathbf{k}}^{k l i}=\mathbf{k}^{k l i}+\mathbf{K}^{k l} \mathbf{t}^{i}, \\
\overline{\mathbf{l}}^{k l i}=\mathbf{l}^{k l i}+\mathbf{L}^{k l} \mathbf{t}^{i}, \\
\overline{\mathbf{f}}=\mathbf{f}^{k}+\mathbf{L}^{k l} \mathbf{t}^{l}, \\
\overline{\mathbf{F}}=\mathbf{F}-\mathbf{L}^{k l} \mathbf{T}^{l k} \mathbf{S} .
\end{gathered}
$$

In terms of these quantities, the residuals take the explicit form,

$$
\begin{gathered}
\mathbf{r}^{i}=\mathbf{f}^{i}-\overline{\mathbf{F}}^{\dagger} \mathbf{t}^{i}+\mathbf{K}\left(\tilde{\mathbf{D}}^{i k}\right)^{k}+\left(2 \mathbf{K}^{i k}-\mathbf{J}^{i k}\right) \mathbf{t}^{k} \\
+\mathbf{S}\left[\tilde{\mathbf{T}}^{i k} \overline{\mathbf{f}}^{k}-\mathbf{T}^{k l} \mathbf{l}^{l k i}-\beta_{i k} \mathbf{t}^{k}\right], \\
\mathbf{R}^{i j}=\mathbf{K}^{i j}+\mathbf{K}\left(\mathbf{D}^{i j}\right)+\alpha_{i j, k l} \mathbf{S D}^{k l} \mathbf{S} \\
+\mathbf{G}^{i j}+\mathbf{G}^{j i \dagger}
\end{gathered}
$$


with

$$
\begin{aligned}
\mathbf{G}^{i j}= & \mathbf{S} \mathbf{T}^{i j} \mathbf{X}+\mathbf{K}\left(\mathbf{E}^{i j}\right)-\beta_{i k} \mathbf{S} \mathbf{T}^{k j} \mathbf{S}, \\
& -\left[\mathbf{K}\left(\mathbf{D}^{i j}\right)^{(k)}+\mathbf{J}^{j k} \mathbf{t}^{i}+\overline{\mathbf{k}}^{i k j}\right] \mathbf{t}^{k \dagger} \mathbf{S} \\
& +\mathbf{S}\left[\tilde{\mathbf{T}}^{i k} \mathbf{Y}^{k j}-\frac{1}{2} \mathbf{T}^{k i} \mathbf{Z}^{k j}-\mathbf{T}^{k j} \mathbf{Z}^{k i}\right] .
\end{aligned}
$$

The intermediates are defined as

$$
\begin{aligned}
& \alpha_{i j, k l}= K_{k l}^{i j}+\operatorname{tr}\left(\mathbf{D}^{i j} \mathbf{K}^{l k}\right)+\mathbf{t}^{i \dagger} \mathbf{k}^{k l j}+\mathbf{t}^{j \dagger} \mathbf{k}^{l k i}, \\
& \beta_{i k}= f_{i k}+\mathbf{f}^{k^{\dagger}} \mathbf{t}^{i}+\operatorname{tr}\left(\mathbf{T}^{i l} \mathbf{L}^{l k}\right)+\mathbf{t}^{l^{\dagger}} \mathbf{l}^{l k i}, \\
& \mathbf{X}=\overline{\mathbf{F}}-\overline{\mathbf{f}}^{k} \mathbf{t}^{k \dagger} \mathbf{S}+\mathbf{G}(\mathbf{E}), \\
& \mathbf{Y}^{k j}=\mathbf{K}^{k j}+\mathbf{K}\left(\mathbf{E}^{k j}\right)-\frac{1}{2}\left[\mathbf{J}^{k j}+\mathbf{J}\left(\mathbf{E}^{k j}\right)\right] \\
&+\left[\frac{1}{4} \mathbf{L}^{k l} \tilde{\mathbf{T}}^{l j}-\frac{1}{2} \overline{\mathbf{l}}^{k l j} \mathbf{t}^{l^{\dagger}}\right] \mathbf{S}, \\
& \mathbf{Z}^{k j}=\mathbf{J}^{k j}+\mathbf{J}\left(\mathbf{E}^{k j}\right)-\left[\frac{1}{2} \mathbf{K}^{l k} \mathbf{T}^{j l}+\overline{\mathbf{k}}^{l k j} \mathbf{t}^{l \dagger}\right] \mathbf{S} .
\end{aligned}
$$

We will finally discuss the approximations made for the terms involving the 3-external integrals. Collecting all 3-external integral contributions gives the following contributions to the singles and doubles residuals:

$$
\begin{gathered}
\Delta r_{s}^{i}=(s u \mid v k) \tilde{D}_{u v}^{i k}, \\
\Delta G_{r s}^{i j}=(s u \mid v k)\left[\delta_{i k} \delta_{v r} t_{u}^{j}-S_{r t}\left(t_{t}^{k} D_{u v}^{i j}-\tilde{T}_{t v}^{i k} t_{u}^{j}\right.\right. \\
\left.\left.+\frac{1}{2} \tilde{T}_{t u}^{i k} t_{v}^{j}+\frac{1}{2} T_{t u}^{k i} t_{v}^{j}+T_{t u}^{k j} t_{v}^{i}\right)\right] .
\end{gathered}
$$

In our program, the set of 3-external integrals $(s u \mid v k)$ depends on domains $u, s, v \in[k]_{3 \mathrm{ext}}$ for each $k$. In most terms, these domains are uniquely determined by the pair classes included in the residual (i.e., $i j, i k$, or $j k$ correspond to strong pairs). The only exception is the term $t_{t}^{k} D_{u v}^{i j}$ in the doubles residual, since here $k$ is not a pair label and, therefore, not limited. However, this term gives only small contributions, and we found it sufficient to restrict $k$ so that the distances $R_{i k}=0$ or $R_{j k}=0$, i.e., the orbital domain of [k] must share at least one atom with $[i]$ or $[j]$. Thus, no additional integrals are needed for these terms. If close pair amplitudes are included in the residuals (option KEEPCLS $=1$ ) the number of 3-external integrals strongly grows. Based on numerical evidence, we found it uncritical to neglect all close-pair contributions that are quadratic in the amplitudes in Eqs. (B27) and (B28). Thus, only the contributions to the singles residual are affected by close pairs, and this leads only to a very minor increase of the number of 3-external integrals.

The required 3-external integral set also depends on the length of the triples list and the size of the triples domains. For each $k$ the united triple domain $[k]_{\mathrm{T}}$ is formed as the union of all triples domains corresponding to the triples containing $k .^{11}[k]_{\mathrm{T}}$ and $[k]_{3 \mathrm{ext}}$ above are then further unified to form the final 3-ext-domains, which, in turn, are used to specify a non-redundant distribution of 3-external integrals in terms of quadruples ( $k R S T$ ) consisting of one LMO index $k$ and three centers (cf. Sec. III B). Increasing the number of close pairs by enlarging IWEAK thus increases $[k]_{\mathrm{T}}$, and with this the size of the 3-external integral distribution.

${ }^{1}$ T. B. Adler and H.-J. Werner, J. Chem. Phys. 135, 144117 (2011).

${ }^{2}$ P. Pulay, Chem. Phys. Lett. 100, 151 (1983).

${ }^{3}$ S. Saeb $\varnothing$ and P. Pulay, Chem. Phys. Lett. 113, 13 (1985).

${ }^{4}$ P. Pulay and S. Saebø, Theor. Chim. Acta 69, 357 (1986).

${ }^{5}$ S. Saeb $\varnothing$ and P. Pulay, J. Chem. Phys. 86, 914 (1987).

${ }^{6}$ S. Saeb $\emptyset$ and P. Pulay, J. Chem. Phys. 88, 1884 (1988).

${ }^{7}$ C. Hampel and H.-J. Werner, J. Chem. Phys. 104, 6286 (1996).

${ }^{8}$ M. Schütz, G. Hetzer, and H.-J. Werner, J. Chem. Phys. 111, 5691 (1999).

${ }^{9}$ M. Schütz and H.-J. Werner, J. Chem. Phys. 114, 661 (2001).

${ }^{10}$ M. Schütz and H.-J. Werner, Chem. Phys. Lett. 318, 370 (2000).

${ }^{11}$ M. Schütz, J. Chem. Phys. 113, 9986 (2000).

${ }^{12}$ M. Schütz, J. Chem. Phys. 116, 8772 (2002).

${ }^{13}$ M. Schütz, Phys. Chem. Chem. Phys. 4, 3941 (2002).

${ }^{14}$ H.-J. Werner, F. R. Manby, and P. Knowles, J. Chem. Phys. 118, 8149 (2003).

${ }^{15}$ M. Schütz, H.-J. Werner, R. Lindh, and F. R. Manby, J. Chem. Phys. 121, 737 (2004).

${ }^{16}$ M. Schütz and F. R. Manby, Phys. Chem. Chem. Phys. 5, 3349 (2003).

${ }^{17}$ H.-J. Werner and K. Pflüger, Ann. Rep. Comp. Chem. 2, 53 (2006).

${ }^{18}$ R. Mata and H.-J. Werner, Mol. Phys. 105, 2753 (2007).

${ }^{19}$ R. Mata, H.-J. Werner, and M. Schütz, J. Chem. Phys. 128, 144106 (2008).

${ }^{20}$ D. Kats, T. Korona, and M. Schütz, J. Chem. Phys. 125, 104106 (2006).

${ }^{21}$ D. Kats, T. Korona, and M. Schütz, J. Chem. Phys. 127, 064107 (2007).

${ }^{22}$ D. Kats and M. Schütz, J. Chem. Phys. 131, 124117 (2009).

${ }^{23}$ K. Freundorfer, D. Kats, T. Korona, and M. Schütz, J. Chem. Phys. 133, 244110 (2010).

${ }^{24}$ Y. Liu and H.-J. Werner (unpublished)

${ }^{25}$ Y. Liu, "A local coupled-cluster method for open-shell molecules: DFLUCCSD(T)," M.S. thesis, University of Stuttgart, 2011.

${ }^{26}$ J. Yang, G. K. L. Chan, F. R. Manby, and H.-J. Werner (unpublished).

${ }^{27}$ J. Yang, Y. Kurashige, F. R. Manby, and G. K. L. Chan, J. Chem. Phys. 134, 044123 (2011).

${ }^{28}$ P. E. Maslen and M. Head-Gordon, Chem. Phys. Lett. 283, 102 (1998).

${ }^{29}$ P. E. Maslen and M. Head-Gordon, J. Chem. Phys. 109, 7093 (1998).

${ }^{30}$ P. Y. Ayala and G. E. Scuseria, J. Chem. Phys. 110, 3660 (1999).

${ }^{31}$ G. E. Scuseria and P. Y. Ayala, J. Chem. Phys. 111, 8330 (1999).

${ }^{32}$ P. E. Maslen, A. Dutoi, M. S. Lee, Y. H. Shao, and M. Head-Gordon, Mol. Phys. 103, 425 (2005).

${ }^{33}$ R. A. DiStasio, Y. S. Jung, and M. Head-Gordon, J. Chem. Theory Comput. 1, 862 (2005).

${ }^{34}$ T. F. Hughes, N. Flocke, and R. J. Bartlett, J. Phys. Chem. A 112, 5994 (2008).

${ }^{35}$ K. V. Lawler, J. A. Parkhill, and M. Head-Gordon, Mol. Phys. 106, 2309 (2008).

${ }^{36}$ T. S. Chwee, A. B. Szilva, R. Lindh, and E. A. Carter, J. Chem. Phys. 128, 224106 (2008).

${ }^{37}$ F. Neese, A. Hansen, and D. G. Liakos, J. Chem. Phys. 131, 064103 (2009).

${ }^{38}$ J. Friedrich and M. Dolg, J. Chem. Theory Comput. 5, 287 (2009).

${ }^{39}$ W. Li, P. Piecuch, J. R. Gour, and S. Li, J. Chem. Phys. 131, 114109 (2009).

${ }^{40}$ M. Ziolkowski, B. Jansik, T. Kjaergaard, and P. Jorgensen, J. Chem. Phys. 133, 014107 (2010).

${ }^{41}$ S. F. Boys and I. Shavitt, Report No. WIS-AF-13, University of Wisconsin, 1959.

42 J. L. Whitten, J. Chem. Phys. 58, 4496 (1973)

${ }^{43}$ E. J. Baerends, D. E. Ellis, and P. Ros, Chem. Phys. 2, 41 (1973).

${ }^{44}$ H. A. Früchtl, R. A. Kendall, R. J. Harrison, and K. G. Dyall, Int. J. Quantum Chem. 64, 63 (1997)

${ }^{45}$ F. Weigend, Phys. Chem. Chem. Phys. 4, 4285 (2002).

${ }^{46}$ R. Polly, H.-J. Werner, F. R. Manby, and P. J. Knowles, Mol. Phys. 102, 2311 (2004).

${ }^{47}$ O. Vahtras, J. Almlöf, and M. W. Feyereisen, Chem. Phys. Lett. 213, 514 (1993).

${ }^{48}$ M. W. Feyereisen, G. Fitzgerald, and A. Komornicki, Chem. Phys. Lett. 208, 359 (1993). 
${ }^{49}$ F. Weigend, M. Häser, H. Patzelt, and R. Ahlrichs, Chem. Phys. Lett. 294, 143 (1998).

${ }^{50}$ F. Weigend, A. Köhn, and C. Hättig, J. Chem. Phys. 116, 3175 (2002).

${ }^{51}$ C. Hättig and F. Weigend, J. Chem. Phys. 113, 5154 (2000).

${ }^{52}$ F. R. Manby, J. Chem. Phys. 119, 4607 (2003).

${ }^{53}$ A. J. May and F. R. Manby, J. Chem. Phys. 121, 4479 (2004).

${ }^{54}$ H.-J. Werner, T. B. Adler, and F. R. Manby, J. Chem. Phys. 126, 164102 (2007).

${ }^{55}$ G. Knizia and H.-J. Werner, J. Chem. Phys. 128, 154103 (2008).

${ }^{56}$ A. P. Rendell and T. J. Lee, J. Chem. Phys. 101, 400 (1994).

${ }^{57}$ L. Maschio, D. Usvyat, F. R. Manby, S. Casassa, C. Pisani, and M. Schütz, Phys. Rev. B 76, 075101 (2007).

${ }^{58}$ M. Schütz, D. Usvyat, M. Lorenz, C. Pisani, L. Maschio, S. Casassa, and M. Halo, in Accurate Condensed-Phase Quantum Chemistry, edited by F. R. Manby (CRC, New York, 2010), pp. 29-55.

${ }^{59}$ H.-J. Werner and F. R. Manby, J. Chem. Phys. 124, 054114 (2006).

${ }^{60}$ F. R. Manby, H.-J. Werner, T. B. Adler, and A. J. May, J. Chem. Phys. 124, 094103 (2006).

${ }^{61}$ T. B. Adler, H.-J. Werner, and F. R. Manby, J. Chem. Phys. 130, 054106 (2009).

${ }^{62}$ S. F. Boys, in Quantum Theory of Atoms, Molecules, and the Solid State, edited by P. O. Löwdin (Academic, New York, 1966), pp. 253-262.

${ }^{63}$ J. Pipek and J. Ladik, Chem. Phys. 102, 445 (1986).

${ }^{64}$ A. E. Reed and F. Weinhold, J. Chem. Phys. 83, 1736 (1985).

${ }^{65}$ B. Jansìk, S. Høst, K. Kristensen, and P. Jørgensen, J. Chem. Phys. 134, 194104 (2011).

${ }^{66}$ P. Pulay, S. Saebø, and W. Meyer, J. Chem. Phys. 81, 1901 (1984).

${ }^{67}$ C. Hampel, K. A. Peterson, and H.-J. Werner, Chem. Phys. Lett. 190, 1 (1992).

68 J. W. Boughton and P. Pulay, J. Comput. Chem. 14, 736 (1993).

${ }^{69}$ A. E. Reed, R. B. Weinstock, and F. Weinhold, J. Chem. Phys. 83, 735 (1985).

${ }^{70}$ A. ElAzhary, G. Rauhut, P. Pulay, and H.-J. Werner, J. Chem. Phys. 108, 5185 (1998)

${ }^{71}$ N. J. Russ and T. D. Crawford, J. Chem. Phys. 121, 691 (2004).

${ }^{72}$ J. E. Subotnik and M. Head-Gordon, J. Chem. Phys. 123, 064108 (2005).

${ }^{73}$ R. Mata and H.-J. Werner, J. Chem. Phys. 125, 184110 (2006).

${ }^{74}$ G. Hetzer, P. Pulay, and H.-J. Werner, Chem. Phys. Lett. 290, 143 (1998).

${ }^{75}$ G. Hetzer, M. Schütz, H. Stoll, and H.-J. Werner, J. Chem. Phys. 113 $9443(2000)$

${ }^{76}$ F. Claeyssens, J. N. Harvey, F. R. Manby, R. A. Mata, A. J. Mulholland, K. E. Ranaghan, M. Schütz, S. Thiel, W. Thiel, and H.-J. Werner, Angew. Chem. 118, 7010 (2006).

${ }^{77}$ R. A. Mata, H.-J. Werner, S. Thiel, and W. Thiel, J. Chem. Phys. 128 025104 (2008)

${ }^{78}$ S. Saebø, W. Tong, and P. Pulay, J. Chem. Phys. 98, 2170 (1993).
${ }^{79}$ M. Schütz, G. Rauhut, and H.-J. Werner, J. Phys. Chem. A 102, 5997 (1998).

${ }^{80}$ B. I. Dunlap, Phys. Chem. Chem. Phys. 2, 2113 (2000).

${ }^{81}$ B. I. Dunlap, J. Mol. Struct.: THEOCHEM 529, 37 (2000).

${ }^{82}$ B. I. Dunlap, J. Mol. Struct.: THEOCHEM 501-502, 221 (2000).

${ }^{83}$ L. Maschio and D. Usvyat, Phys. Rev. B 78, 073102 (2008).

${ }^{84}$ C. Pisani, L. Maschio, S. Casassa, M. Halo, M. Schütz, and D. Usvyat, J. Comput. Chem. 29, 2113 (2008).

${ }^{85}$ H.-J. Werner, P. J. Knowles, G. Knizia, F. R. Manby, M. Schütz et al., Molpro, version 2010.1, a package of ab initio programs, see http://www.molpro.net.

${ }^{86}$ H.-J. Werner, P. J. Knowles, G. Knizia, F. R. Manby, and M. Schütz, "Molpro: a general-purpose quantum chemistry program package," Comput. Mol. Sci. (in press).

${ }^{87}$ G. Rauhut and H.-J. Werner, Phys. Chem. Chem. Phys. 5, 2001 (2003).

${ }^{88}$ T. Hrenar, G. Rauhut, and H.-J. Werner, J. Phys. Chem. A 110, 2060 (2006)

${ }^{89}$ T. Korona, K. Pflüger, and H.-J. Werner, Phys. Chem. Chem. Phys. 6, 2059 (2004).

${ }^{90}$ J. M. Dieterich, H.-J. Werner, R. A. Mata, S. Metz, and W. Thiel, J. Chem. Phys. 132, 035101 (2010)

${ }^{91}$ T. B. Adler, "Local explicitly correlated methods for the treatment of large molecules close to the basis set limit," M.S. thesis, University of Stuttgart, 2011.

${ }^{92}$ K. Sadeghian, M. Bocola, and M. Schütz, ChemPhysChem 12, 1251 (2011).

${ }^{93}$ T. H. Dunning, Jr., J. Chem. Phys. 90, 1007 (1989).

${ }^{94}$ R. A. Kendall, T. H. Dunning, and R. J. Harrison, J. Chem. Phys. 96, 6796 (1992).

${ }^{95}$ M. Schütz, R. Lindh, and H.-J. Werner, Mol. Phys. 96, 719 (1999).

${ }^{96}$ A. Karton and J. Martin, Theor. Chem. Acc. 115, 330 (2006).

${ }^{97}$ T. Helgaker, W. Klopper, H. Koch, and J. Noga, J. Chem. Phys. 106, 9639 (1997).

${ }^{98}$ T. B. Adler, G. Knizia, and H.-J. Werner, J. Chem. Phys. 127, 221106 (2007)

${ }^{99}$ G. Knizia, T. B. Adler, and H.-J. Werner, J. Chem. Phys. 130, 054104 (2009).

${ }^{100}$ D. E. Brash, J. A. Rudolph, J. A. Simon, A. Lin, G. J. McKenna, H. P. Baden, A. J. Halperin, and J. Pontén, Proc. Natl. Acad. Sci. U.S.A. 88, 10124 (1991)

${ }^{101}$ A. Sancar, Chem. Rev. 103, 2203 (2003).

${ }^{102}$ In our previous papers, (Refs. 7, 9, and 67) the quantities $D_{r s}^{i j}$ were denoted as $C_{r s}^{i j}$. In those papers, $D_{r s}^{i j}$ also included additional contributions $\delta_{i r} t_{s}^{j}$ $+\delta_{j s} t_{r}^{i}$. Here we changed the notation because the extended $D_{r s}^{i j}$ matrices are not needed, and $C_{r s}^{i j}$ is used in F12-theory in another context, see Ref. 1. 